\title{
Diabetes increases mortality after myocardial infarction by oxidizing CaMKII
}

\author{
Min Luo, ${ }^{1}$ Xiaoqun Guan, ${ }^{1}$ Elizabeth D. Luczak, ${ }^{1}$ Di Lang, ${ }^{2}$ William Kutschke, ${ }^{1}$ Zhan Gao, ${ }^{1}$ \\ Jinying Yang, ${ }^{1}$ Patric Glynn, ${ }^{3}$ Samuel Sossalla, ${ }^{4}$ Paari D. Swaminathan, ${ }^{1}$ \\ Robert M. Weiss, ${ }^{1}$ Baoli Yang, ${ }^{5}$ Adam G. Rokita, ${ }^{1,4}$ Lars S. Maier, ${ }^{4}$ \\ Igor R. Efimov, ${ }^{2}$ Thomas J. Hund, ${ }^{3}$ and Mark E. Anderson ${ }^{1,6}$
}

\begin{abstract}
${ }^{1}$ Division of Cardiovascular Medicine, Department of Internal Medicine, Cardiovascular Research Center, Carver College of Medicine, University of lowa, Iowa City, lowa, USA. ${ }^{2}$ Department of Biomedical Engineering, Washington University, St. Louis, Missouri, USA. ${ }^{3}$ Department of Biomedical Engineering, The Ohio State University, Columbus, Ohio, USA. ${ }^{4}$ Department of Cardiology, University of Goettingen, Goettingen, Germany. ${ }^{5}$ Gene Targeting Core Facility and Department of Obstetrics and Gynecology, University of lowa, lowa City, lowa, USA. ${ }^{6}$ Department of Molecular Physiology and Biophysics, University of lowa Carver College of Medicine, lowa City, Iowa, USA.
\end{abstract}

\begin{abstract}
Diabetes increases oxidant stress and doubles the risk of dying after myocardial infarction, but the mechanisms underlying increased mortality are unknown. Mice with streptozotocin-induced diabetes developed profound heart rate slowing and doubled mortality compared with controls after myocardial infarction. Oxidized $\mathrm{Ca}^{2+} /$ calmodulin-dependent protein kinase II (ox-CaMKII) was significantly increased in pacemaker tissues from diabetic patients compared with that in nondiabetic patients after myocardial infarction. Streptozotocin-treated mice had increased pacemaker cell ox-CaMKII and apoptosis, which were further enhanced by myocardial infarction. We developed a knockin mouse model of oxidation-resistant CaMKII $\delta$ (MM-VV), the isoform associated with cardiovascular disease. Streptozotocin-treated MM-VV mice and WT mice infused with MitoTEMPO, a mitochondrial targeted antioxidant, expressed significantly less ox-CaMKII, exhibited increased pacemaker cell survival, maintained normal heart rates, and were resistant to diabetes-attributable mortality after myocardial infarction. Our findings suggest that activation of a mitochondrial/ox-CaMKII pathway contributes to increased sudden death in diabetic patients after myocardial infarction.
\end{abstract}

\section{Introduction}

Diabetes mellitus is a worldwide epidemic and a major public health problem that affects over $8 \%$ of the US population $(1,2)$. Myocardial infarction (MI) is the most common cause of mortality in diabetics $(3,4)$, and multiple studies have shown that diabetic patients are twice as likely to die from MI compared with nondiabetic patients $(2,4-6)$. However, the mechanism(s) for the "diabetic factor" (7) underlying increased MI-related mortality in diabetic patients is unclear. Surprisingly, the excess mortality due to MI in diabetic patients is independent of commonly recognized comorbid clinical conditions, including the extent of myocardial injury, left ventricular contractile dysfunction, or coronary artery patency after reperfusion therapy $(4-6,8)$. Improved understanding of molecular mechanisms and pathways that promote death in diabetic patients after MI is a major goal of biomedical science.

ROS are elevated after MI $(9,10)$, and increased ROS promotes disease complications of diabetes $(11,12)$. However, broad-spectrum antioxidant therapies have yielded disappointing results (13), suggesting that detailed knowledge of oxidative injury mechanisms will be necessary to develop new and effective targeted antioxidant therapies. The multifunctional $\mathrm{Ca}^{2+} /$ calmodulin-dependent $^{2}$ protein kinase II (CaMKII) is activated by oxidation (ox-CaMKII) (14), and ox-CaMKII may increase the risk of sudden death after

Conflict of interest: Mark E. Anderson is a named inventor on intellectual property claiming to treat myocardial infarction by CaMKII inhibition and is a cofounder of Allosteros Therapeutics, a biotech company aiming to develop enzyme-based therapies.

Citation for this article: J Clin Invest. 2013;123(3):1262-1274. doi:10.1172/JCI65268
MI by promoting heart failure (15), cardiac rupture (16), and arrhythmias (17). We found significantly more ox-CaMKII in diabetic hearts compared with that in nondiabetic hearts in patients who had MI, suggesting that ox-CaMKII could contribute to the increased mortality in diabetic patients after MI. Streptozotocin (STZ) is a pancreatic $\beta$ cell toxin that induces a severe form of type I diabetes (18), and STZ-treated diabetic mice were twice as likely to die after MI surgery as vehicle-treated control mice, mimicking the increased mortality in diabetic patients compared with that in nondiabetic patients after MI. Oxidation of methionines 281/282 in the CaMKII regulatory domain lock ox-CaMKII into a constitutively active conformation (14). In order to test whether ox-CaMKII was an essential component of a molecular pathway that increased death in STZ-treated mice after MI, we created a knockin model of oxidation-resistant CaMKII by replacing the methionines 281/282 with valines (MM-VV) in a myocardial CaMKII isoform (CaMKIIס) known to participate in myocardial injury $(19,20)$. Diabetic, STZtreated MM-VV mice and mice with transgenic myocardial and sinoatrial nodal (SAN) pacemaker cell expression of a synthetic CaMKII inhibitory peptide (AC3-I) (21) were protected from increased mortality after MI, indicating that increased ox-CaMKII was essential for excess mortality after MI in STZ-treated mice.

Death in STZ-treated mice after MI was due to severe bradycardia, consistent with known defects in cardiac pacemaker function in diabetic patients (22-24) and recognized associations between abnormal cardiac pacing and increased risk for sudden death (25). The increase in SAN ox-CaMKII expression in diabetes is likely a result of increased mitochondrial ROS triggered by hyperglycemia, as diabetic mice treated with a mitochondrial antioxidant, MitoTEMPO, showed reduced ox-CaMKII, preserved heart rates, and improved survival 
A

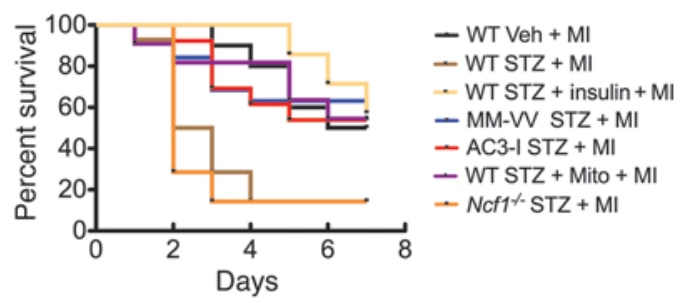

C
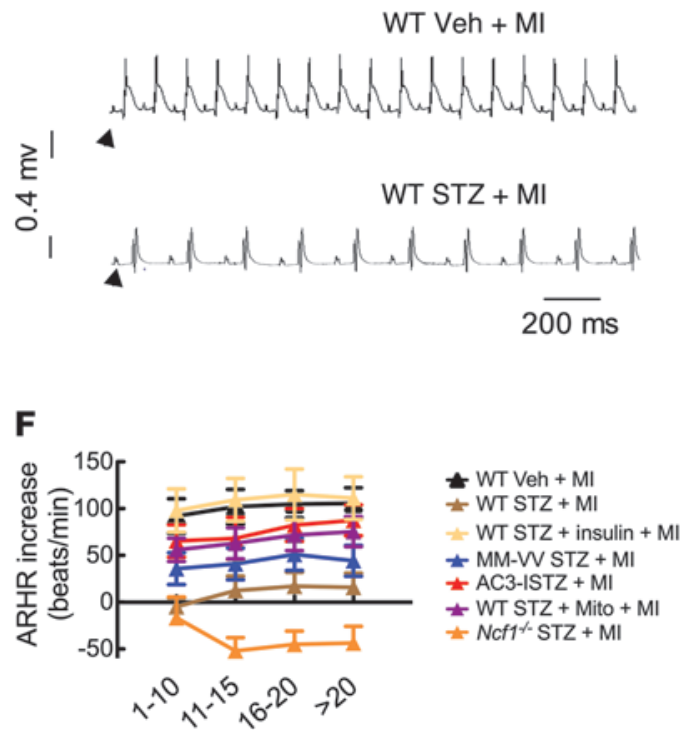

B

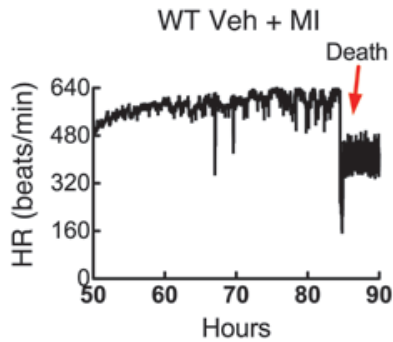

D

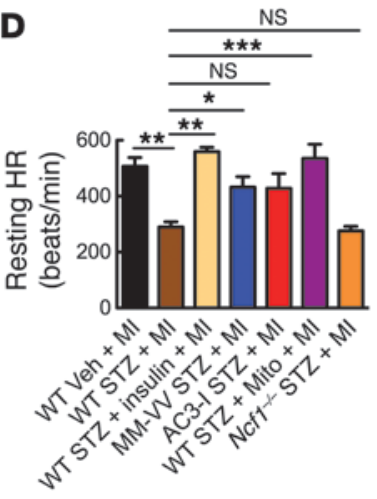

WT STZ + MI
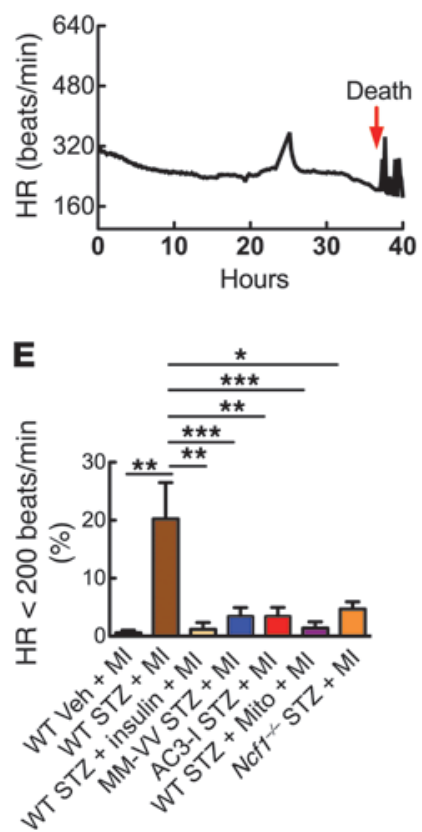

Figure 1

Increased death and SND in WT diabetic mice after MI. (A) Survival in WT mice treated with vehicle (Veh) $(n=10)$, STZ $(n=14)$, STZ and insulin $(n=7)$, and MitoTEMPO (Mito) and STZ $(n=11)$ and in AC3-I $(n=13)$, MM-VV $(n=19)$, and Ncf1-- mice treated with STZ $(n=7)$ after MI. Overall $P=0.005$ by 1 -way ANOVA, $P<0.05$ for comparisons between WT STZ and all other groups except the Ncf1-- group $(P=0.7)$. (B-F) In vivo data from ECG-telemetered WT mice treated with vehicle, STZ, STZ and insulin, and MitoTEMPO and STZ and from AC3-I, MM-VV, and Ncf1-/- mice treated with STZ after MI ( $n=4-14$ per group). (B) Representative heart rate (HR) tracings ending in death. (C) Representative ECGs. Arrowheads indicate $P$ waves. (D) Resting heart rates. Overall $P<0.0001,{ }^{*} P<0.05,{ }^{* \star} P<0.01,{ }^{* \star} P<0.001$ by 1-way ANOVA. (E) Episodes of severe bradycardia (heart rate $<200$ beats per minute). Overall $P=0.0004,{ }^{\star} P<0.05$, ${ }^{\star \star} P<0.01,{ }^{* * *} P<0.001$ by 1 -way ANOVA. (F) Spontaneous activity-responsive heart rate (ARHR) increase (overall $P=0.001$ for activity $1-10, P=0.0005$ for activity level $11-15, P=0.0002$ for all other activity levels by 1 -way ANOVA).

after MI. Our findings are consistent with a pathway in which hyperglycemia enhances susceptibility to mortality after MI by increasing mitochondrial ROS, leading to excessive ox-CaMKII, SAN cell apoptosis, SAN dysfunction, and death. These results provide new insights into a novel mechanism underlying increased mortality in diabetes and $\mathrm{MI}$ and suggest that mitochondrial or CaMKII-targeted antioxidant therapies could benefit high-risk diabetic patients.

\section{Results}

Diabetes increases MI mortality by SAN injury. STZ-treated mice had over a 2-fold increase in mean blood glucose compared with that of controls (Supplemental Figure 1; supplemental material available online with this article; doi:10.1172/JCI65268DS1). Two to three weeks after STZ treatment, we performed MI surgery in STZ-injected or vehicle-injected mice by ligating the left anterior descending coronary artery $(15,16,21)$. Similar to diabetic patients (4-6), diabetic mice (WT) had significantly decreased survival after MI compared with that of nondiabetic mice (Figure 1A). However, there was no difference in survival after sham surgery between diabetic and nondiabetic mice (Supplemental Figure 2A), suggesting that the increased mortality in diabetic mice was due to MI. There were also no significant changes in serum chemistry (i.e., blood urea nitrogen or bicarbonate) to suggest ketoacidosis or acute renal failure as a potential cause of excessive mortality in STZ-treated mice (Supplemental Table 1). Most early deaths after MI are caused by heart failure due to depressed mechanical function (26), arrhythmias (27), or myocardial rupture (28). To investigate the cause of the excessive acute death in diabetic mice after MI, we performed autopsies on all dead mice and found that 4 out of 5 deaths in nondiabetic mice were caused by cardiac rupture. In contrast, no rupture events were found in any STZ-treated diabetic mice. We performed echocardiography $(2.1 \pm 0.3$ days in vehicle and $2.0 \pm 0.4$ days in STZ group after MI) and found that there were no differences in left ventricular contractile function, chamber dilation (Supplemental Table 2), or in heart weight and lung weights normalized to tibia length between the STZ-treated or control groups 
A
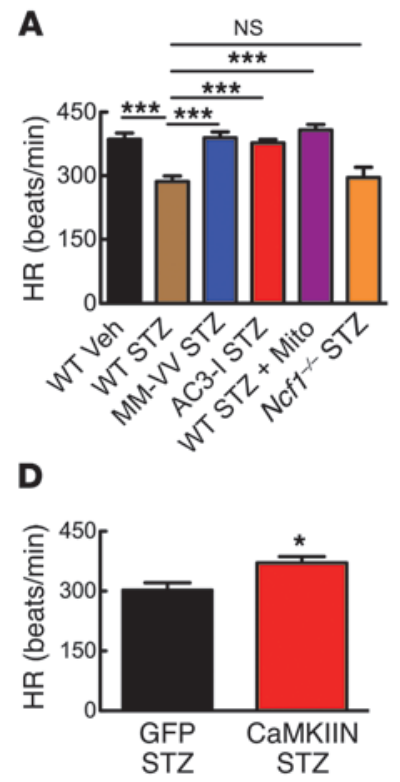

B
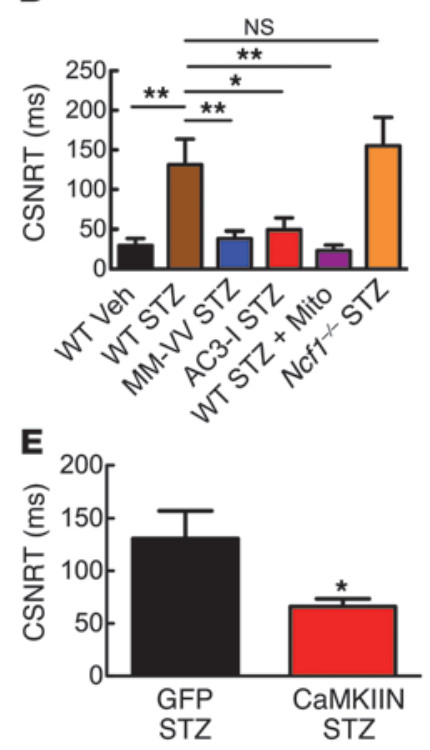

C
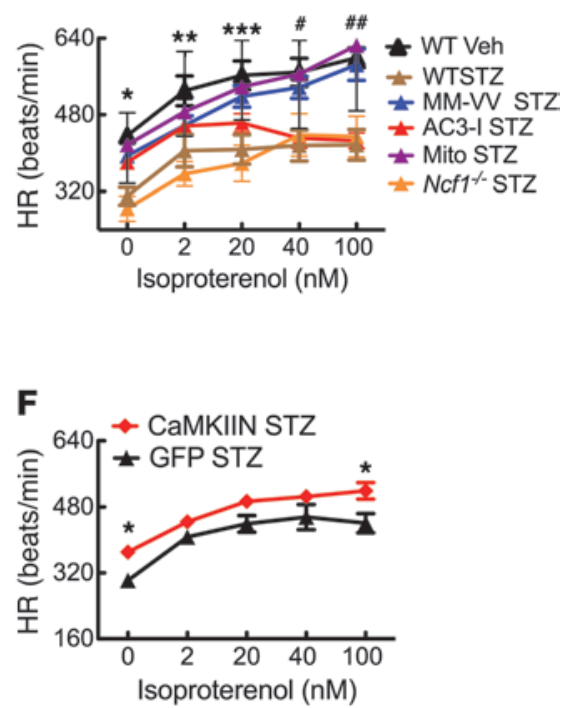

\section{Figure 2}

Intrinsic SND in diabetic mice. Langendorff-perfused hearts from WT mice treated with vehicle, STZ, and MitoTEMPO and STZ and AC3-I, MM-VV, and $\mathrm{Ncf1}^{-1-}$ mice treated with STZ. (A) Heart rate. Overall $P<0.0001,{ }^{\star \star \star} P<0.001$ by 1 -way ANOVA $(n=6-15$ per group). (B) CSNRT. Overall $P=0.0003,{ }^{* *} P<0.01,{ }^{*} P<0.05$ by 1 -way ANOVA $\left(n=5-8\right.$ per group). (C) Response to isoproterenol. ${ }^{\star} P<0.0001$ for 0 nM, ${ }^{* \star} P=0.0009$ for $2 \mathrm{nM},{ }^{* * \star} P=0.0001$ for $20 \mathrm{nM},{ }^{\#} P=0.002$ for $40 \mathrm{nM}, \# \#<<0.0001$ for $100 \mathrm{nM}(n=5-8$ per group). (D-F) Langendorff-perfused hearts isolated from STZ-treated mice after SAN painting with adenovirus expressing CaMKIIN and GFP or GFP alone. (D) Heart rates $\left({ }^{\star} P=0.01 ; n=6-7\right.$ per group). (E) CSNRT ( ${ }^{*} P=0.04 ; n=6$ per group). (F) Responses to isoproterenol $(P>0.05$ for all doses excluding $100 \mathrm{nM}$, ${ }^{\star} P<0.05 ; n=6-7$ per group).

(Supplemental Table 1). There were also no significant differences in cardiac output $(16 \pm 1.5 \mathrm{ml} / \mathrm{min}$ in vehicle and $14 \pm 0.7 \mathrm{ml} / \mathrm{min}$ in STZ group; $P=0.1)$ or left ventricular ejection fraction $(76 \% \pm 2.5 \%$ in vehicle and $76 \% \pm 1.4 \%$ in STZ group; $P=0.8$ ) prior to $\mathrm{MI}$ in diabetic mice compared with nondiabetic mice. These results suggest that intrinsic defects in the contractile function of surviving left ventricular myocardia of STZ-treated mice were not the primary cause of increased mortality after MI. However, echocardiographic studies did show that diabetic mice had decreased heart rates without a significant change in left ventricular stroke volume, which caused significantly $(P=0.019)$ reduced cardiac output compared with that of the nondiabetic mice (Supplemental Table 2). These data pointed to a defect in SAN function as a potential determinant of increased mortality after MI in diabetic mice.

After MI, diabetic patients are at increased risk for sudden death due to cardiac arrhythmias $(6,29,30)$, disorders of myocardial cell membrane excitability that result in excessively fast or slow heart rates. In order to investigate whether arrhythmia contributes to the excessive death in diabetic mice after MI, we continuously monitored heart rate and rhythm in unrestrained and unanesthetized mice surgically implanted with ECG- and activitysensing telemeters. We measured ECGs immediately after recovery from MI for up to 1 week or until death. Diabetic mice had marked heart rate slowing throughout the recording period, but heart rates slowed profoundly prior to death (Figure 1B, right). In contrast, deaths in vehicle-treated control mice after MI were not preceded by heart rate slowing (Figure 1B, left). The severe heart rate slowing was accompanied by reduced heart rate variability in diabetic mice compared with that in nondiabetic mice after MI
(Figure 1B), similar to findings in high-risk diabetic patients (22, 23). Analysis of the ECG traces showed slow heart rates emanating from the SAN, presumably due to defective SAN function, were the predominant cause of severe heart rate slowing (Figure 1C). WT diabetic mice after MI exhibited a significant reduction in heart rate at rest compared with that of vehicle-treated MI controls (Figure 1D) and suffered frequent episodes of severe heart rate slowing, below 200 beats per minute, after MI (Figure 1E). In contrast, heart rate slowing events below 200 beats per minute were rarely detected in vehicle-treated mice after MI (Figure 1E). Furthermore, WT diabetic mice after MI had reduced heart rate responses to activity compared with those of vehicle-treated control mice after MI (Figure 1F), whereas both groups showed a similar distribution of spontaneous activity throughout the recording period (Supplemental Figure 3). We did not identify episodes of abnormal rapid rhythms in any of the mice. These findings are consistent with features of severe SAN dysfunction (SND), slow resting heart rates and inadequate activity-related increases in heart rate. These findings are also consistent with known defects in cardiac pacemaker and autonomic nerve function in diabetic patients that are thought to cause reduced heart rate variability and prevent activity-related increases in heart rate $(22,23,31)$. Thus, we interpreted our findings up to this point to suggest that SND, due to defects in SAN cells, autonomic activity, or both, contributes to increased mortality after MI in diabetic mice.

In vivo heart rate is determined by intrinsic SAN automaticity under extrinsic modulation by autonomic nerves (32). In order to examine the mechanism of SND, we first asked whether STZinduced diabetes in the absence of MI was sufficient to cause SND 
A

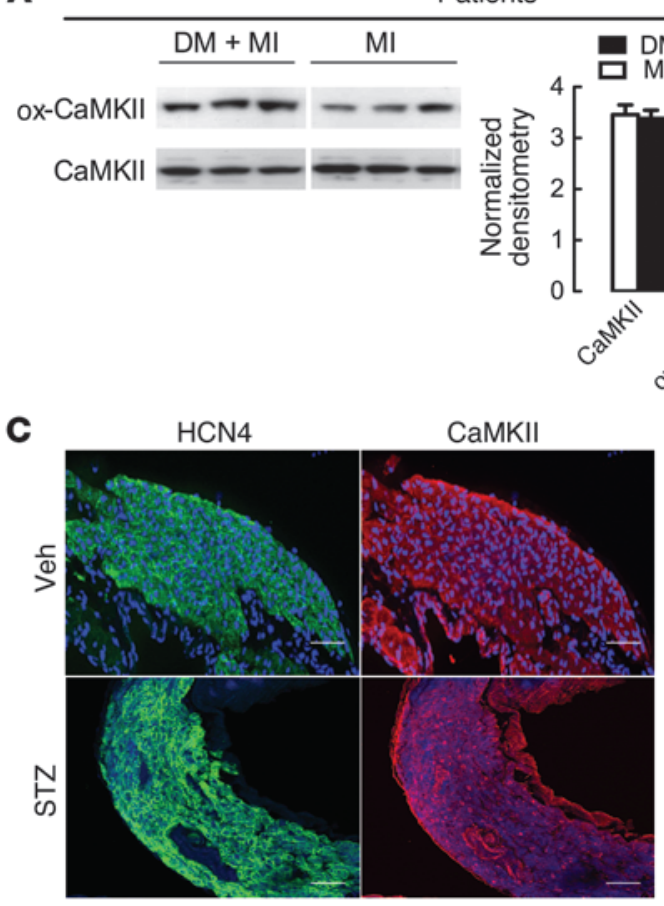

B

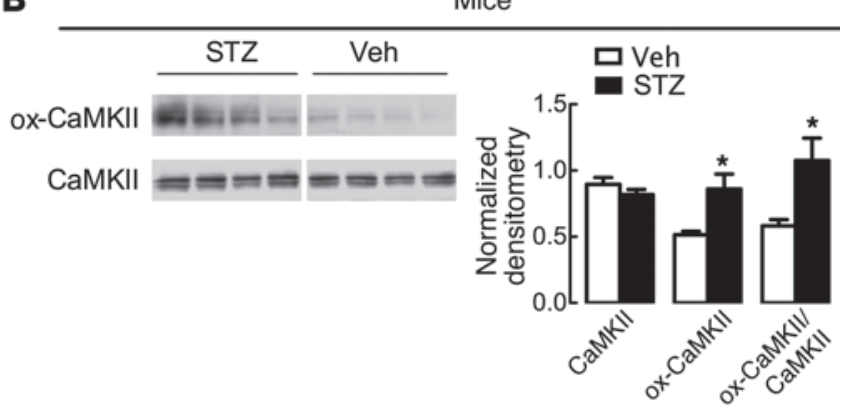

D

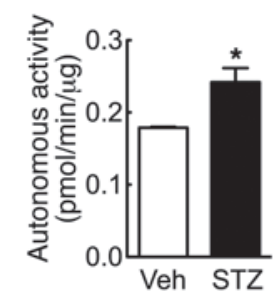

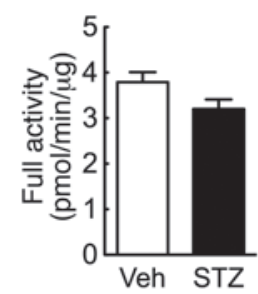

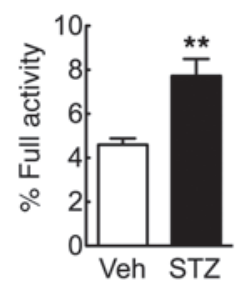

$\mathrm{HCN} 4$

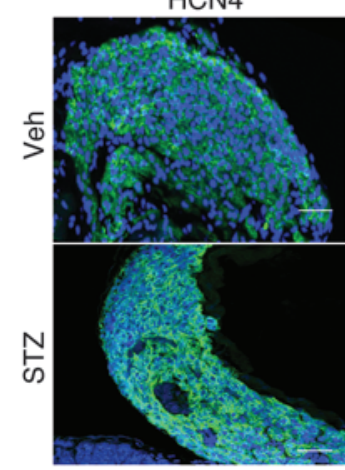

ox-CaMKII

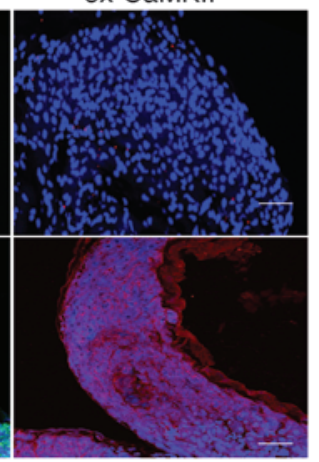

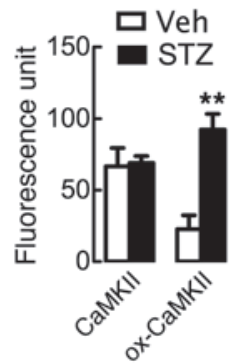

Figure 3

Increased ox-CaMKII in diabetic patients and mice. (A) Representative immunoblots from right atrial tissues obtained from patients with or without diabetes mellitus and with MI. Band intensity was normalized to Coomassie staining. Summary data show CaMKII $(P=0.7)$, ox-CaMKII ( $\left.{ }^{\star} P=0.02\right)$, and ox-CaMKII/CaMKII ( $\left.{ }^{* *} P=0.03\right)$ in patients with diabetes mellitus (DM) and MI $(n=5)$ and MI alone $(n=5)$. Lanes were run on the same gel but were noncontiguous. (B) Representative immunoblots from right atria tissue obtained from STZ- or vehicle-treated mice. Summary data show CaMKII $(P=0.3)$, ox-CaMKII ( $\left.{ }^{*} P=0.04\right)$, and ox-CaMKII/CaMKII ( $\left.{ }^{\star} P=0.04\right)$ in STZ- $(n=5)$ and vehicle-treated mice $(n=4)$. Lanes were run on the same gel but were noncontiguous. Band intensity was normalized to Coomassie staining. (C) Representative immunofluorescence images in SAN from mice treated with vehicle or STZ. HCN4 (green) marks SAN, and DAPI (blue) marks nuclei. Scale bars: $50 \mu m$. Summary data show CaMKII $(P=0.8)$ and ox-CaMKII $\left({ }^{* *} P=0.005\right)$ in STZ- $(n=5)$ and vehicle-treated mice $(n=3)$. (D) Ca ${ }^{2+} /$ calmodulin-independent autonomous activity $\left({ }^{\star} P=0.03\right.$, left), full activity $\left(P=0.08\right.$, middle), and autonomous/full activity $\left({ }^{* *} P=0.01\right.$, right) in hearts from STZ- $(n=6)$ and vehicle-treated mice $(n=4)$.

and whether SND was evident under conditions in which autonomic regulation was absent. We measured heart rates in ex vivo, Langendorff-perfused hearts and in atrial explants that contained the SAN region isolated from diabetic and nondiabetic mice. Heart rates were significantly slower in WT diabetic hearts compared with those in nondiabetic counterparts (Figure 2A). We measured the sinus node recovery time corrected for heart rate (CSNRT), a clinical index of SAN function (33), in the Langendorff-perfused hearts. The CSNRT was significantly prolonged in WT diabetic mice compared with that in nondiabetic mice (Figure 2B). Furthermore, hearts isolated from WT STZ-treated mice also showed impaired responses to isoproterenol (Figure 2C).

An advantage of the STZ model of type I diabetes is that it allowed us to focus on hyperglycemia as an upstream cause of increased mortality after MI. In order to test whether the increase in mortality and SND in our STZ and MI model was specifically due to hyperglycemia, we treated WT STZ and MI mice with insulin, which corrected STZ-induced hyperglycemia (Supplemental Figure 1). We confirmed that increased mortality after MI required hyperglycemia, because mortality after MI was reduced to control levels by insulin replacement (Figure 1A). WT STZ-treated mice rescued by exogenous insulin were resistant to SND, showing improved resting (Figure 1D) and activity-induced heart rate increases (Figure $1 \mathrm{~F})$. These findings suggest that hyperglycemia alone is sufficient to increase mortality after MI and show that potential offtarget actions of STZ are unlikely to contribute to SND.

ox-CaMKII is increased in diabetic patients and STZ-treated mice. Increased ROS is a consistent feature of diabetes and is thought to contribute to diabetic heart disease (11). We recently identified CaMKII as an oxidant sensor (15-17) and found that excessive SAN 

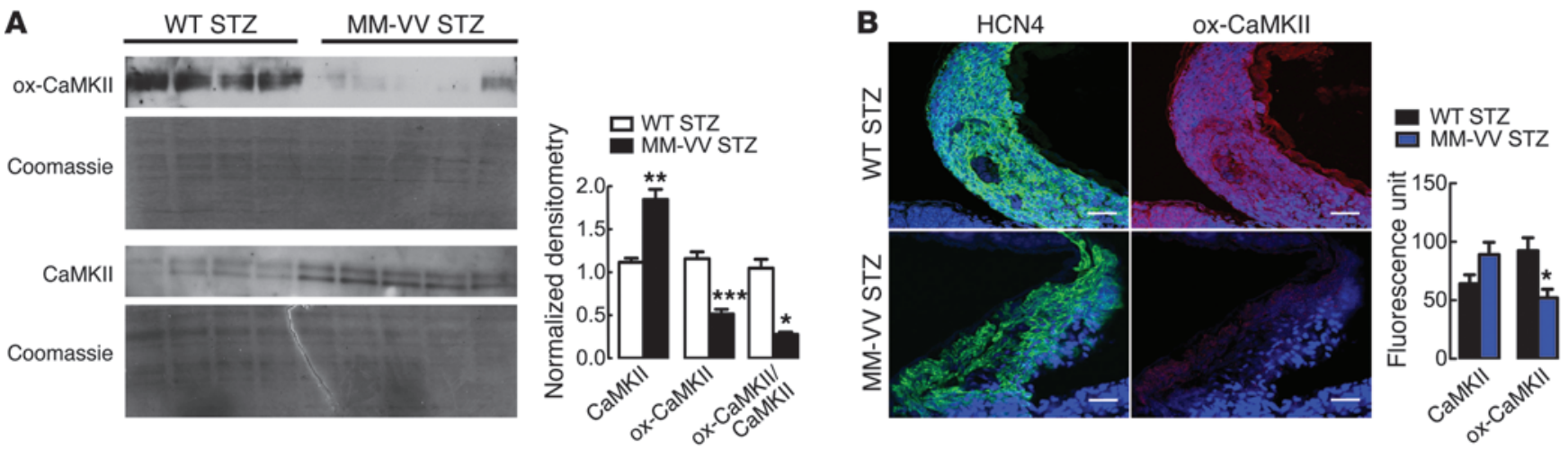

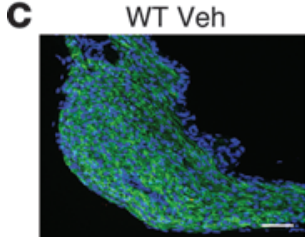

MM-VV STZ
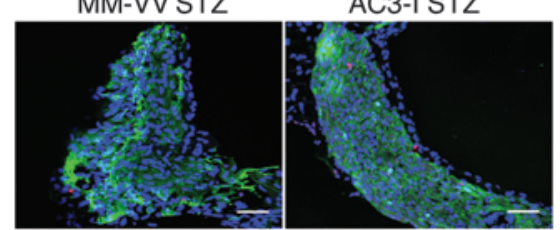

WT STZ

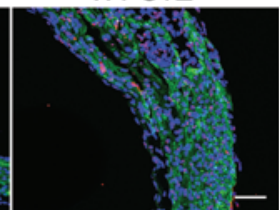

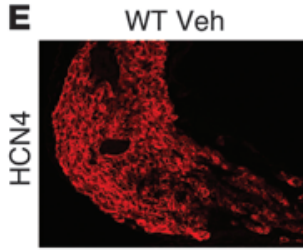

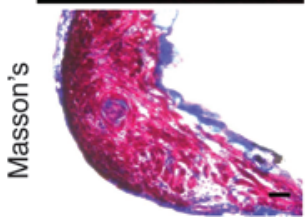

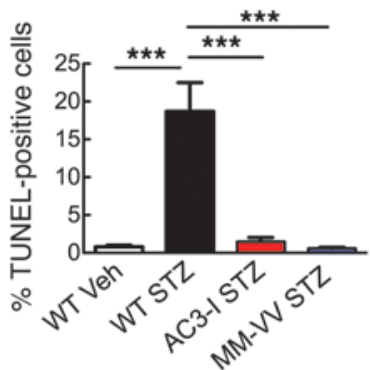

D

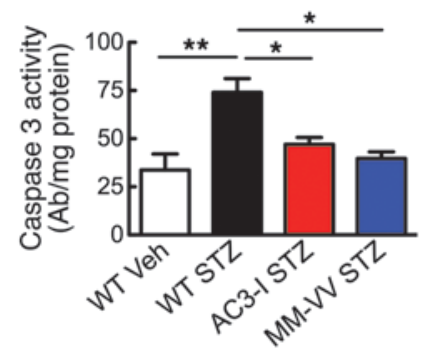

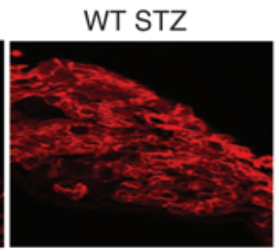

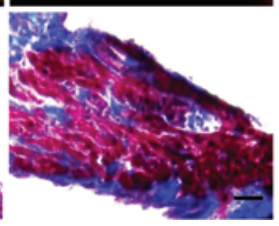

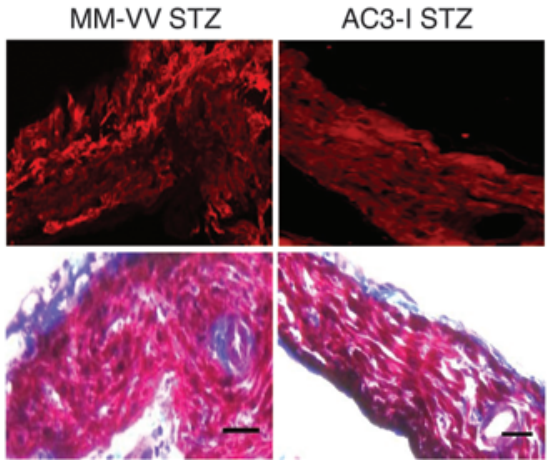

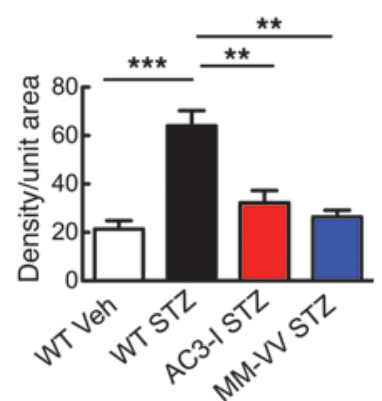

\section{Figure 4}

Oxidation-resistant CaMKII and myocardial CaMKII inhibition protects against SAN cell death and fibrosis. (A) Representative immunoblots and Coomassie-stained gels from right atria of STZ-treated MM-VV and WT mice. CaMKII ( $\left.{ }^{* *} P=0.001\right)$, ox-CaMKII $\left.{ }^{* \star *} P \leq 0.001\right)$, and ox-CaMKII/ CaMKII ( $\left.{ }^{*} P=0.002\right)$ in MM-VV $(n=5)$ and WT STZ-treated mice $(n=4)$. (B) Representative immunofluorescence images of SAN from MM-VV and WT STZ-treated mice. Scale bars: $50 \mu \mathrm{m}$. CaMKII $(P=0.09)$ and ox-CaMKII ( $\left.{ }^{*} P=0.02\right)$ in MM-VV $(n=4)$ and WT STZ-treated mice $(n=5)$. HCN4 (green) marks SAN, and DAPI (blue) marks nuclei. (C) Representative SAN sections show TUNEL-positive staining (72) in WT mice after vehicle or STZ treatment and in MM-VV and AC3-I mice after STZ treatment. DAPI (blue) marks nuclei, HCN4 (green) marks SAN. Scale bars: 50 um. Overall $P<0.0001,{ }^{* * *} P<0.001$ by 1-way ANOVA ( $n=3-5$ per group). (D) Caspase-3 activity in SAN from WT mice after vehicle or STZ treatment and in MM-VV and AC3-I mice after STZ treatment. Overall $P=0.007,{ }^{\star} P<0.05$, ${ }^{\star *} P<0.01$ by 1 -way ANOVA $(n=3$ per group). (E) Representative Masson's trichrome staining for fibrosis in SAN from STZ-treated WT, MM-VV, and AC3-I mice. Scale bars: $20 \mu \mathrm{m}$. Overall $P=0.005$, ${ }^{* \star} P<0.01$, ${ }^{* * *} P<0.001$ by 1 -way ANOVA ( $n=3-4$ per group).

ox-CaMKII was associated with SND in patients and caused SND in mice infused with angiotensin II (17). Based on the apparent relationship between ox-CaMKII and SND, we next asked whether ox-CaMKII was elevated in myocardia from diabetic patients and mice. We measured ox-CaMKII using an antiserum against oxidized methionines (methionines 281/282) $(15,17)$ from right atrial samples, nearby to the SAN. We found significantly increased expression of ox-CaMKII in myocardia from patients with a history of MI and diabetes compared with that in patients with a history of MI but without diabetes (Figure 3A and Supplemental Table 3). Similarly, ox-CaMKII was significantly increased in right atrial and SAN tissues from STZ-treated diabetic mice compared with those from nondiabetic mice (Figure 3, B and C). In contrast to the changes in ox-CaMKII, total right atrial CaMKII expression was 
A ${ }_{1.8}$ Mathematical model of intact sinoatrial node

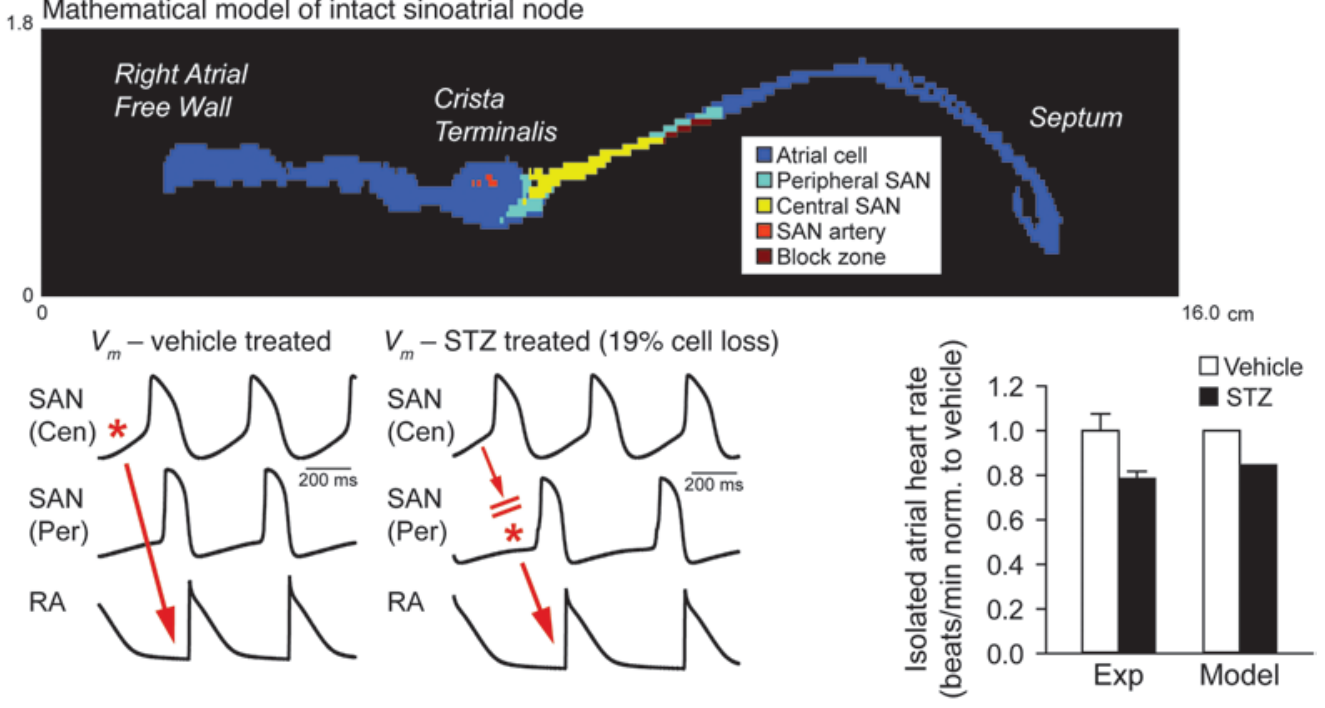

B
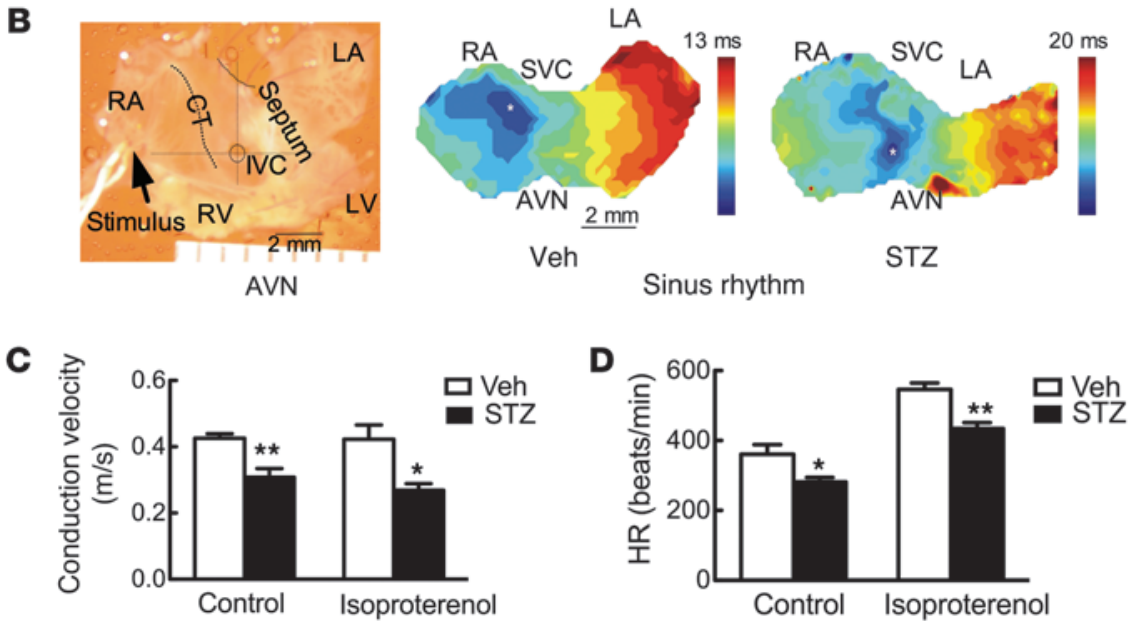

\section{Figure 5}

SAN cell death and fibrosis lead to decreased conduction velocity and spontaneous beating. (A) Cell loss data from vehicle- and STZ-treated WT mice were incorporated into a 2-dimensional histologically reconstructed mathematical model of the intact sinoatrial node (action potentials from central [Cen] and peripheral [Per] SAN and right atrium are shown). The STZ model with 19\% cell loss in the SAN predicts a shift of lead pacemaker site (red asterisks on action potential traces), slowing of conduction time and block (double red lines on action potential traces), and decreased heart rate similar to experimental measurements. Red arrows denote direction of action potential propagation. (B) Mouse atria prepared for optical measurements (left). Original magnification, $\times 20$. Representative pseudocolored isochrones from optical mapping (middle and right). SVC, superior vena cava; AVN, atrioventricular node; IVC, inferior vena cava; LA, left atrium; RA, right atrium; LV, left ventricle; RV, right ventricle; CT, crista terminalis. White asterisks denote the earliest activation in the SAN. (C) Conduction velocity during pacing in STZ- and vehicle-treated mice in the absence $\left({ }^{* *} P=0.004 ; n=5\right.$ per group) and presence of isoproterenol ( $3 \mathrm{nM},{ }^{*} P=0.01 ; n=5$ per group). (D) Heart rate in isolated atria in the absence ( ${ }^{\star} P=0.03 ; n=4-5$ per group) or presence of isoproterenol (ISO; $3 \mathrm{nM},{ }^{* *} P=0.0056 ; n=4-5$ per group).

equivalent in patients with or without diabetes and in diabetic and nondiabetic mice (Figure 3, A-C), suggesting that diabetes predominantly affects CaMKII by posttranslational activation. ox-CaMKII is constitutively active, even in the absence of $\mathrm{Ca}^{2+} /$ calmodulin (15), so we next tested whether increased ox-CaMKII resulted in more $\mathrm{Ca}^{2+} /$ calmodulin-autonomous CaMKII activity. As expected, the cardiac lysates from STZ-treated diabetic mice had significantly increased $\mathrm{Ca}^{2+}$ - and calmodulin-independent autonomous CaMKII activity compared with vehicle-treated control hearts (Figure 3D). These findings show that ox-CaMKII is increased in diabetic hearts from patients and mice. The parallel increases in ox-CaMKII in myocardial tissues from diabetic mice and patients suggest that oxCaMKII may contribute to high mortality rates in diabetic patients who suffer from MI, potentially by inducing SND.

STZ-treated mice had SND but did not die spontaneously over the time course of our study, suggesting that MI may trigger more extensive SND by enhancing SAN oxidation. To test this idea, we measured SAN oxidation in STZ- and vehicle-treated mice with and without MI. We found that STZ treatment alone markedly increased SAN ROS, as detected by the ROS fluorescent indicator dihydroethidium (DHE), while MI approximately doubled the intensity of DHE fluorescence and ox-CaMKII in STZ-treated 
A
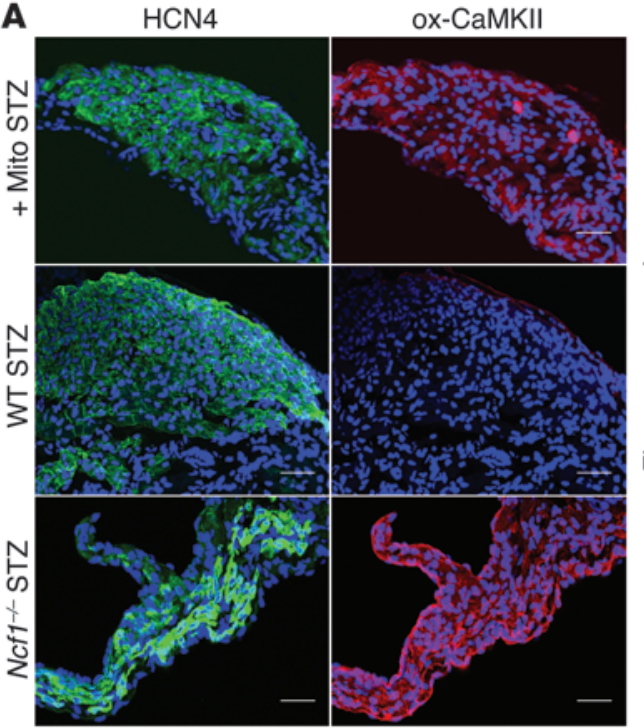

\section{(15)}

WT STZ

WT STZ + Mito

$\mathrm{Ncf1}^{-1} \mathrm{STZ}$
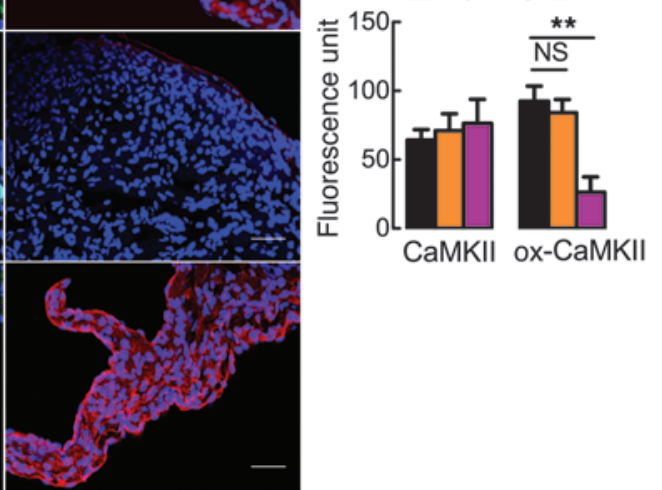

B

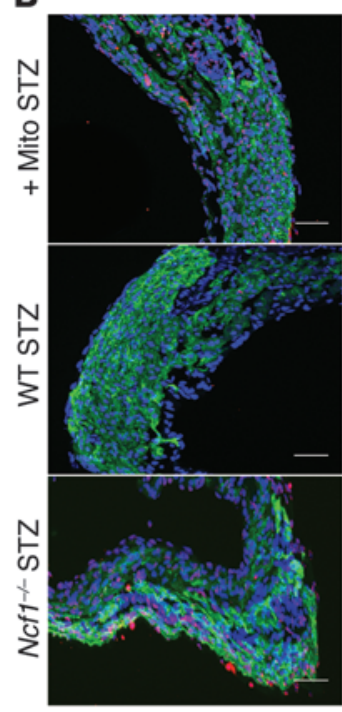

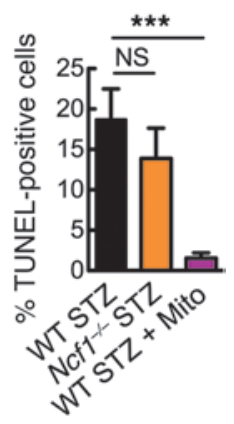

C

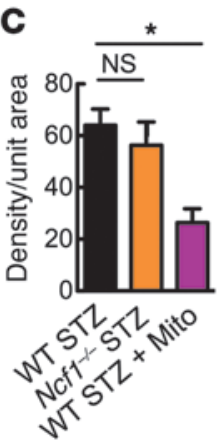

D

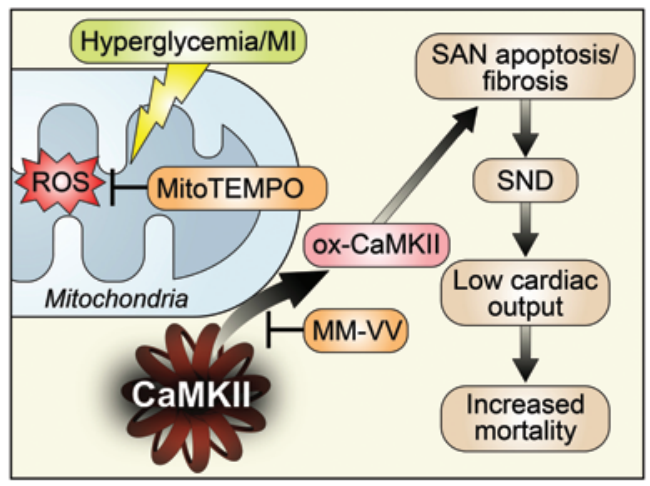

Figure 6

Mitochondrial ROS increases ox-CaMKII and SAN cell death. (A-C) SAN staining from WT mice treated with STZ alone and MitoTEMPO and STZ and $\mathrm{Ncf1}^{-1-}$ mice treated with STZ. DAPI (blue) marks nuclei, HCN4 (green) marks SAN. Scale bars: 50 um. (A) Representative sections show oxCaMKII expression. Overall $P=0.006,{ }^{*} P<0.01$ by 1 -way ANOVA $(n=3-5$ per group). (B) Representative sections show TUNEL-positive cells. Overall $P=0.0006,{ }^{* * *} P<0.01$ by 1 -way ANOVA ( $n=3-7$ per group). (C) Fibrosis measured by Masson's trichrome staining. Overall $P=0.016,{ }^{*} P<0.05$ by 1-way ANOVA ( $n=3-4$ per group). (D) Proposed model for CaMKII activation by STZ-induced diabetes. STZ-induced hyperglycemia and MI promote mitochondrial ROS generation, which activates ox-CaMKII to cause SND, leading to increased mortality after MI.

mice compared with vehicle-treated mice (Supplemental Figure 4, A and B), suggesting that MI and diabetes independently promote oxidation in SAN.

Oxidation-resistant CaMKII eliminates increased mortality from STZ after MI. The MM-VV mutation in CaMKIIS selectively disables oxidative activation of CaMKII without affecting normal $\mathrm{Ca}^{2+} / \mathrm{calm}^{-}$ odulin-dependent CaMKII activation or the transition to $\mathrm{Ca}^{2+} /$ calmodulin-independent activation by threonine 287 autophosphorylation (15). The MM-VV mice (Supplemental Figure 5) had an increase in blood glucose after STZ treatment (Supplemental Figure 1) and an increase in ROS in right atrial tissues, as measured by DHE (Supplemental Figure 6), that was equivalent to that in WT STZ-treated mice, but MM-VV STZ-treated mouse hearts had significantly less ox-CaMKII expression compared with STZtreated WT controls in right atrial tissues (Figure 4A) and SAN (Figure 4B). Although MM-VV mice without STZ treatment had similar survival after MI compared with WT mice (Supplemental Figure 2B), the MM-VV diabetic mice were significantly resistant to increased mortality after MI compared with WT diabetic controls (Figure 1A), despite similar left ventricle contractile function and volumes after MI compared with WT diabetic mice (Supplemental Figure 7). The MM-VV STZ-treated mice were resistant to SND after MI, with improvement in resting heart rate (Figure 1D), reduced episodes of very low heart rate ( $<200$ beats per minute) (Figure 1E), and improved activity-related heart rate increases (Figure 1F) compared with STZ-treated WT controls after MI. The distribution of spontaneous activity was similar between MM-VV and WT diabetic mice after MI (Supplemental Figure 3), further suggesting that improved survival in MM-VV mice was not due to generally improved well-being or enhanced myocardial function. We next measured the effects of STZ treatment without MI on SAN function, in the absence of autonomic innervation, using Langendorff-perfused hearts isolated from MM-VV and WT control mice. Hearts from the STZ-treated MM-VV diabetic mice showed improvement in intrinsic sinus node function, as measured by increased spontaneous heart rate (Figure 2A), decreased CSNRT 
(Figure 2B), and improved heart rate responses to isoproterenol (Figure 2C), compared with WT diabetic mice. These results show that selective ablation of methionines $281 / 282$ on CaMKIIS is sufficient to protect against STZ-induced oxidant SAN injury and to indicate that oxidation of CaMKII $\delta$ is required for excess mortality in mice with diabetes after MI.

CaMKII inbibition protects against SND and mortality. The MM-VV knockin mutation occurs in all tissues expressing CaMKIIס, so it is possible that the improved SAN function and improved survival in these mice were due to unanticipated extra-myocardial effects of oxidation-resistant CaMKIII. With this thought in mind, we next asked whether cardiac-selective CaMKII inhibition was sufficient to prevent increased mortality and SND in STZ-treated mice after MI. We subjected transgenic mice with myocardial- (21) and SAN-delimited (17) transgenic expression of AC3-I, a synthetic peptide with inhibitory activity against all CaMKII isoforms, to MI surgery after STZ or vehicle injection. Similar to the MM-VV mice, the AC3-I diabetic mice had significant improvement in survival after MI (Figure 1A), without showing evidence of improved left ventricle contractile function or different left ventricular volumes compared with those of WT diabetic mice (Supplemental Figure 7). These findings suggest that improved survival was not due to improvement in left ventricular structure or function. AC3-I diabetic mice were also resistant to $\mathrm{SND}$, as shown by improvement in resting heart rate (Figure 1D), reduced episodes of severe heart rate slowing (Figure $1 \mathrm{E}$ ), and increased activity-responsive increases in heart rate (Figure 1F), after MI compared with WT diabetic mice after MI. We next examined SAN function in STZ-treated AC3-I and WT control hearts in the absence of MI. Langendorff-perfused hearts isolated from AC3-I diabetic mice showed improvement in intrinsic sinus node function, as measured by increased spontaneous heart rates (Figure 2A) and decreased CSNRT (Figure 2B), compared with those from WT diabetic mice. The response in heart rate to isoproterenol in AC3-I hearts was blunted (Figure 2C), as expected based on the role of CaMKII to promote $\beta$ adrenergic receptor agonist increases in heart rate (34). These results suggest that myocardial- and SAN-selective CaMKII inhibition protects against SND and excessive mortality after $\mathrm{MI}$ in diabetic mice.

We next asked whether SAN-targeted CaMKII inhibition was sufficient to prevent SND in the absence of "global" cardiac CaMKII inhibition. We used a SAN-targeted gene transfer technique (17) to deliver the most potent and selective known biological CaMKII inhibitor, CAMKIIN, a peptide that is endogenously expressed in brain but not in heart (35). Adenovirus encoding CaMKIIN and EGFP or an adenovirus encoding EGFP alone was painted on SAN of WT mice treated with STZ. Langendorff-perfused hearts isolated from diabetic mice after SAN painting with adenovirus expressing CaMKIIN and EGFP, compared with those painted with adenovirus expressing EGFP alone, showed improved resting heart rate (Figure 2D) and reduced CSNRT (Figure 2E). Similar to that in AC3-I hearts, the response in heart rate to isoproterenol in mice with SAN CaMKIIN expression was blunted (Figure 2F). These data confirm that SAN-targeted CaMKII inhibition protects against diabetes-induced SND.

Diabetes causes SND by CaMKII-triggered SAN cell apoptosis and fibrosis. Cardiomyocyte apoptosis and ROS are increased in diabetes but the molecular mechanism for connecting increased oxidant stress to diabetes-mediated apoptosis is uncertain (36, 37). Excessive ox-CaMKII activity also promotes apoptosis, leading to mal- adaptive cardiovascular responses to injury, including SND (15, 17). The SAN consists of a small group of highly specialized pacemaker cells that reside in the lateral right atrium. The relatively small number of cells in the SAN, compared with the larger number of contracting myocardial cells, make physiological SAN function particularly vulnerable to SAN cell death; SND occurs when SAN cell loss exceeds a threshold value, leading to a source-sink mismatch between SAN and surrounding atrial myocardium (17). We found significantly increased TUNEL staining and caspase-3 activity, indicating increased cell death, in right atrial tissues containing SAN (Figure 4, C and D) from diabetic mice compared with those from nondiabetic mice. In contrast, MM-VV and AC3-I mice were resistant to diabetes-induced increases in TUNEL staining and caspase-3 activity (Figure 4, C and D). MM-VV and AC3-I mice treated with STZ and subjected to MI were protected from SAN apoptosis compared with WT controls (Supplemental Figure 8). These findings are consistent with a concept that SND arises from STZ and MI as a form of oxidant injury that requires SAN ox-CaMKII-mediated SAN cell death.

SAN tissue from patients with SND is also marked by increased fibrosis and destruction of normal architecture that may contribute to conduction slowing and defective impulse transmission $(32,38)$. In order to test whether diabetes causes SAN fibrosis characteristic of patients with SND, we quantified SAN and atrial collagen by Masson's trichrome staining. Collagen expression within the SAN was higher in diabetic mice compared with that in nondiabetic mice (Figure 4E). In contrast, MM-VV and AC3-I diabetic mice did not exhibit increased fibrosis (Figure 4E). Taken together, these data show that diabetes promotes ox-CaMKII, SAN cell apoptosis, fibrosis, and SND.

In order to quantify the contribution of SAN cell death to SND in our STZ and MI model, we incorporated measured values for SAN cell loss from nondiabetic and diabetic mice into a 2-dimensional computational model of SAN, based on histologically reconstructed right atrial geometry (17). Normal activation of the atrial myocardium (vehicle treated in Figure 5A) was triggered by a regular impulse that initiates in the central SAN. Our mathematical model revealed that increased cell loss in diabetic hearts greatly disrupted the normal capture of atrial myocardium by SAN impulses. Specifically, the model with increased cell loss displayed a shift of the lead pacemaker site toward the SAN periphery, a delay in conduction time from the SAN to the atrial myocardium, and slowing of heart rate comparable to experimentally measured values (Figure 5A and Supplemental Videos 1 and 2). Thus, our mathematical model of the intact SAN predicts that cell loss alone can produce SND characterized by slowed heart rate. We next measured spontaneous impulse formation in current-clamped SAN cells isolated from diabetic and vehicle-infused control mice. SAN cells from both groups showed similar spontaneous automaticity at baseline and in response to isoproterenol (Supplemental Figure 9), suggesting that SAN cell loss in STZtreated mice, rather than defects in the function of surviving SAN cells, was the primary cause of STZ-induced SND. Thus, computational and experimental analyses support a view that excessive SAN cell death is a fundamental event causing increased mortality after MI in the setting of diabetes.

Patients with SND exhibit widespread atrial electrophysiological abnormalities secondary to destruction of normal SAN architecture, leading to slowed conduction velocity (32). To determine whether STZ-induced diabetes slowed conduction velocity, we 
performed potentiometric optical mapping in isolated atria from STZ- and vehicle-treated mice. To control for differences in intrinsic heart rate between STZ- and vehicle-treated mice, we measured conduction velocity in isolated atria during fixed rate stimulation. We found significantly decreased conduction velocity in STZtreated mice compared with that in control mice in the absence and presence of isoproterenol (Figure 5C). In addition, heart rates were significantly slower in isolated atria from diabetic hearts compared with nondiabetic counterparts (Figure 5D). Furthermore, atria isolated from STZ-treated mice also showed impaired responses to isoproterenol (Figure 5D). Taken together, these findings support a view that STZ treatment resulted in SND that was caused, at least in part, by intrinsic defects in SAN function. Our optical mapping studies (Figure 5, B and C) and computational modeling (Figure 5A) also support the concept that atrial myocardium is injured by STZ treatment, as evidenced by decreased conduction velocity measured throughout the atrial tissues.

Mitochondria are the source of ROS for increasing ox-CaMKII in diabetes and MI. Diabetes is known to increase ROS production in mitochondria and apoptosis in cardiomyocytes $(11,36,37)$, but to our knowledge mitochondrial ROS generation has not been linked to SND in diabetes. To determine whether diabetes causes SND by enhancing mitochondrial ROS generation and SAN ox-CaMKII, we implanted mice with osmotic mini-pumps eluting MitoTEMPO $(700 \mu \mathrm{g} / \mathrm{kg} / \mathrm{d})$, a mitochondrial targeted ROS scavenger with generalized antioxidant activities, including superoxide dismutase $(39,40)$, immediately prior to STZ treatment. The ROS levels measured by DHE (Supplemental Figure 10) and ox-CaMKII levels were significantly reduced in SAN from MitoTEMPO-treated mice compared with those in control diabetic mice (Figure 6A). MitoTEMPO-treated diabetic mice had significantly fewer TUNEL-stained SAN cells (Figure 6B) and decreased fibrosis compared with control diabetic mice (Figure $6 \mathrm{C}) \cdot \mathrm{p} 47$ phox encodes a cytosolic regulatory subunit of NADPH oxidase, a multicomponent ROS-generating enzyme complex. Angiotensin II, aldosterone, and MI all increase ox-CaMKII, at least in part, by activating a $47^{\text {phox }}$ containing NADPH oxidase (15-17). In contrast, STZ treatment does not appear to increase ox-CaMKII by activating NADPH oxidase, because $\mathrm{Ncf1^{-/- }}$ ( $p 47^{\text {phox- }- \text { - }) ~ S T Z-t r e a t e d ~ m i c e ~ w e r e ~ n o t ~ p r o t e c t e d ~ f r o m ~ i n c r e a s e s ~}$ in ox-CaMKII or SAN cell apoptosis and fibrosis after MI (Figure 6, A-C). MitoTEMPO-treated diabetic mice, but not $\mathrm{Ncf1}^{-{ }^{--}}$diabetic mice, had improved survival after MI (Figure 1A), despite similar left ventricle contractile function and chamber volumes after MI compared with WT diabetic mice (Supplemental Figure 7). MitoTEMPO-treated diabetic mice, but not $\mathrm{Ncf1}^{-/-}$diabetic mice, were also resistant to SND, as evidenced by improvement in heart rate at rest (Figure 1D), significantly decreased episodes of heart rate $\leq 200$ beats per minute (Figure $1 \mathrm{E}$ ), and enhanced heart rate response to activity (Figure 1F) after MI compared with WT diabetic mice. We examined the effects of STZ treatment without MI in the absence of autonomic innervation using Langendorff-perfused hearts isolated from MitoTEMPO- and STZ-treated WT mice and STZ-treated $\mathrm{Ncf1}^{-/-}$mice. MitoTEMPO infusion improved heart rates (Figure 2A), normalized CSNRT (Figure 2B), and restored control level heart rate responses to isoproterenol (Figure 2C) compared with WT diabetic and $\mathrm{Ncf1} 1^{-/-}$diabetic mice. In contrast, $\mathrm{Ncf1}^{-{ }^{-}}$diabetic mice had heart rate responses similar to those of WT diabetic mice (Figure 2C). We interpret these data to indicate that mitochondrial gener- ated ROS is required for diabetes-induced ox-CaMKII elevation, increased SAN cell death, and SND and to suggest that multiple, injury-specific sources of ROS have the potential to oxidize CaMKII and induce pathological consequences in heart.

Based on the requirement for increased glucose for SND phenotypes (Figure 1, D-F), we next used cultured neonatal myocytes to determine whether elevated glucose was sufficient to increase mitochondrial ROS and/or apoptosis. Neonatal myocytes responded to high glucose $(33 \mathrm{mM})$, a concentration similar to the elevated plasma glucose levels in diabetic mice, with increased ROS and apoptosis (Supplemental Figure 11). In contrast, neither a lower glucose concentration $(5 \mathrm{mM})$ nor mannitol $(33 \mathrm{mM})$, a metabolically inert glucose congener, increased ROS or apoptosis. ROS and apoptosis were reduced by MitoTEMPO (1 mM) (Supplemental Figure 11). Neonatal cardiomyocytes isolated from MM-VV mice responded to high glucose treatment with increased ROS but not apoptosis (Supplemental Figure 11). These findings support the hypothesis that hyperglycemia in diabetic mice stimulates mitochondrial ROS production, leading to apoptosis.

\section{Discussion}

Elevated ROS is a consistent finding in cardiovascular diseases, but understanding of defined molecular targets and pathways that connect increased oxidation with disease phenotypes is limited $(11,14,38,41)$. Here we show that selective ablation of oxidative activation of CaMKII $\delta$ was sufficient to eliminate the increased risk of sudden death in diabetic mice after MI. The hearts from MM-VV diabetic mice exhibited a normal heart rate response to isoproterenol, showing that oxidation of CaMKII is not required for fight-or-flight physiological responses and suggesting that $\beta$ adrenergic receptor activation was not a decisive factor in oxidative injury for STZ-treated mice after MI. Our MM-VV mutant mouse only targeted the CaMKII isoform. The finding that ox-CaMKII $\delta$-resistant MM-VV mice were not susceptible to STZ-induced SND or increased mortality after MI is potentially consistent with recent studies showing that $\mathrm{CaMKII \delta ^{-/- }}$ mice with residual CaMKII activity, presumably due to CaMKII $\delta$-alternative CaMKII isoforms, were nevertheless resistant to cardiac injury (19, 20). Our studies identified SAN as a critical target of a novel and unanticipated oxidation-sensitive molecular pathway underlying increased mortality after MI in diabetic mice. This ox-CaMKII pathway may also increase mortality risk in diabetic patients, who show more severe heart rate defects consistent with $\operatorname{SND}(22,23$, 31) and higher right atrial ox-CaMKII than nondiabetic patients after MI. Because multiple tissues are injured in diabetes, it will be important to learn if ox-CaMKII contributes to progression of diverse forms of diabetic injury, such as diabetic vasculopathy and neuropathy. It is intriguing that CaMKII inhibition was recently found to improve insulin sensitivity in diabetes (42), suggesting that CaMKII inhibition could benefit diabetic patients by multiple mechanisms and pathways.

Increased rates of sudden cardiac death after MI in diabetic patients are due to arrhythmia $(4,6,8,30)$, and some data suggest excessively slow heart rates contribute to this excess mortality. For example, among the 41,021 patients of the GUSTO-I cohort, a study demonstrating that diabetic patients had increased mortality after acute MI despite contemporary thrombolysis, the rates of bradycardia were consistently higher among diabetic patients (6). Furthermore, loss of heart rate variability, a marker of SAN and autonomic nerve function that is defective in diabetic patients, 
positively correlates with increased acute mortality after MI (43). Although reduced heart rate variability, a feature of SND, could be due to intrinsic SAN defects, loss of normal autonomic function, or a combination of both, our ex vivo findings indicate that STZ treatment directly affects SAN, causing intrinsic SND by increased apoptosis, fibrosis, and subsequent electrical source-sink mismatch between SAN and myocardium. Our findings do not dispute a role for autonomic dysfunction in diabetes-induced defects in heart rate but appear consistent with other evidence showing diabetes causes pathological effects on SAN (44-46).

Our findings suggest that SAN injury induced by hyperglycemia sensitizes the SAN to MI-induced dysfunction, as evidenced by profoundly slow heart rates, low cardiac output, and death. Our study suggests that MI aggravates SND by further increasing ROS, ox-CaMKII, and apoptosis of SAN cells in diabetic mice. We conclude that increased acute mortality after MI in diabetic mice is primarily caused by SND, but our findings do not exclude the possibility of other contributing factors. For example, left ventricular end diastolic volumes in our diabetic mice did not increase, despite increased filling time as a result of profound bradycardia (Supplemental Table 2), suggesting that STZ-treated mice after MI have diastolic dysfunction, a recognized feature of diabetic cardiomyopathy (47). Furthermore, MI activates a multitude of neurohumoral factors, including the renin-angiotensin system, which could also contribute to SND (17).

Clinical trials have shown that optimal glucose management reduces mortality from cardiovascular diseases in diabetic patients $(48,49)$. However, the more recent ACCORD study reported conflicting results, which indicated that intensive glucose lowering was not beneficial for cardiovascular mortality (50). Nevertheless, meta-analysis on major randomized trials, including the ACCORD study, concluded that intensive control groups $(0.9 \% \mathrm{~A} 1 \mathrm{C}$ reduction over 5 years) significantly reduced events of nonfatal MI by $17 \%$ and coronary heart disease by $15 \%$ (51). It appears that the glucose level itself, not the form of glucose intolerance, may be the key driver of mortality risk in diabetic patients suffering from MI. In support of this view is a meta-analysis report in which nondiabetic patients presenting with hyperglycemia had a similar risk of mortality as that for diabetic patients after MI (52). Our studies showed that increased diabetes-attributable mortality in STZ-treated mice was prevented after insulin replacement, demonstrating that hyperglycemia rather than off-target actions of STZ caused increased mortality after MI. Our study used an STZ-induced model of type 1 diabetes with severe hyperglycemia. This model is limited, as it lacks the metabolic complexity of type 2 diabetes, a condition that represents the majority of the diabetic population. The hyperglycemia level in STZ-treated mice may not mimic the levels in the diabetic population exactly. Nevertheless, the STZ model of type 1 diabetes allows a clear focus on the role of hyperglycemia, which is the key metabolic alteration in both type 1 and type 2 diabetes.

Despite of the availability of insulin therapy, glycemic control remains a major challenge in diabetes management $(53,54)$. Inadequate glycemic control likely accounts for the high prevalence of diabetic complications, such as MI, and the associated significant morbidity and mortality. Hyperglycemia is common in patients with MI and tends to persist throughout the early period after infarction (55-57). For example, in a study of approximately 30,000 patients admitted to hospitals with acute MI, about half of the patients were hyperglycemic and had significantly higher mor- tality early after MI compared with their normoglycemic counterparts $(52,56)$. We interpret the data from our human specimens to show that increased ox-CaMKII may be a feature of human diabetes in the setting of MI but recognize that our data set is not adequately powered for subgroup analysis. We also acknowledge the limitations of this study using patient samples with an antecedent MI in the context of focusing on ox-CaMKII in mice in the periMI setting. Nevertheless, our observations suggest that increased ox-CaMKII may be a marker of poor glycemic control in patients with coronary artery disease. Therefore, it is possible that the benefits of glucose control are due, at least in part, to reduction in ox-CaMKII.

Increased oxidative stress brought about by hyperglycemia is an important link between diabetes and its cardiac complications $(11,12)$. Until now, the cellular source of ROS known to oxidize CaMKII was NADPH oxidase (15-17). Here we show that STZ treatment increased ox-CaMKII by augmenting mitochondrial ROS, apparently independent of NADPH oxidases. Increased glucose in vivo, due to STZ treatment, or in vitro was required to increase ROS, while infused MitoTEMPO effectively suppressed hyperglycemia-induced ROS, SND, and sudden death. Mitochondria constitutively generate superoxide radicals as a by-product of electron transport and are also important sources for intracellular ROS production triggered by hyperglycemia $(58,59)$. Our current findings, taken together with earlier studies (15-17), suggest that CaMKII is available for oxidation by multiple pathways. The finding that myocardia from STZ-treated MM-VV mice had equivalent increases in ROS compared with controls shows that ox-CaMKII $\delta$ is downstream to ROS for causing SND and increased mortality after MI. However, CaMKII $\gamma$ has been implicated in enhancing mitochondrial $\mathrm{Ca}^{2+}$, a process that could potentially promote mitochondrial ROS generation (60), and we recently found that CaMKII increased mitochondrial $\mathrm{Ca}^{2+}$ entry by activating the mitochondrial $\mathrm{Ca}^{2+}$ uniporter (61). Thus, it is possible that CaMKII contributes to ROS generation after STZ treatment, functioning as an "upstream" and "downstream" signal in oxidative injury. The trend toward reduced STZ-induced ROS in AC3-I mice (Supplemental Figure 6) is potentially consistent with this concept, because AC3-I inhibits all CaMKII isoforms. We used AC3-I mice to compare the effects of global myocardial CaMKII inhibition with MM-VV mice that are selectively protected from oxidative activation of CaMKIIס.

We originally identified ox-CaMKII in response to angiotensin II stimulation of myocardium (15). The renin-angiotensin system is activated in diabetes, and angiotensin II inhibition was shown to be even more beneficial in improving mortality after MI in diabetic patients than in nondiabetic patients $(62,63)$. Both angiotensin II antagonists $(62,63)$ and anti-hyperglycemic therapy (51) improve mortality in diabetic patients after MI. These data from clinical trials raise the issue of whether some of the benefits of validated, conventional therapies for diabetes work by reducing ox-CaMKII. Our findings are consistent with the idea that ox-CaMKII could be a "diabetic factor" and suggest that CaMKII inhibition or antioxidant therapy targeted to CaMKII or mitochondria could be effective preventive therapies for diabetic patients at high risk for MI.

\section{Methods}

Mouse models. We performed our studies on male and female mice with C57BL/ 6 backgrounds at approximately 3-5 months of age. Mice lacking the $\mathrm{Ncf1}$ gene ( $\mathrm{Ncf1}^{-/-}$mice) were purchased from The Jackson Laboratory. Mice with transgenic myocardial CaMKII inhibition (AC3-I mice) were 
generated by us, as previously described (21). MM-VV mice were generated as follows (Supplemental Figure 5): the 2 homologous arms, left arm (LA, containing exons 9-12) and right arm (RA, containing exon 13), were directly amplified via high-fidelity PCR using the genomic DNA prepared from a murine ES cell strain W4 (129/SvEvTac). The mutations were created in exon 11 of LA. The LA and RA were cloned into OSDupDel.Neo, a generic targeting vector containing both Lox-P-flanked Neo cassette (MC1-Neo) and thymidine kinase cassette (PGK-TK) to obtain a final targeting construct. The targeting construct linearized with NotI was electroporated into W4 cells. After selection with G418, targeted homologous replacement recombinants were identified by specific PCRs and by DNA sequencing. The confirmed ES cell lines were microinjected into C57BL/6J blastocysts (Uteri transfer) to obtain chimeras that were crossed with C57BL/6J to verify germ line transmission. The transmitted germ lines were mated with EIIa-Cre Tg mice (C57BL/6J, The Jackson Laboratory) to obtain final allele with Neo cassette removal. These heterozygote mice (64) were bred to obtain homozygote knockin mice identified by PCR genotyping using tail DNA.

STZ-induced diabetic model. After overnight fast, mice were injected intraperitoneally with streptozocin (150 mg/kg, Sigma-Aldrich) dissolved in a mix of citrate buffer (citric acid and sodium citrate, $\mathrm{pH} 4.8$ ) or vehicle (citrate buffer). Blood glucose was checked 3-5 days later via tail vein; mice with a blood glucose level above $300 \mathrm{mg} / \mathrm{dl}$ were considered diabetic. For a subset of animals, long acting insulin (Lantus) was injected subcutaneously every 24 hours 3-5 days after STZ treatment to maintain normoglycemia.

$M I$ and ECG and activity telemetry. Mice were anesthetized with ketamine/ xylazine $(87.5 / 12.5 \mathrm{mg} / \mathrm{kg})$, and left anterior descending artery was ligated with 8-0 suture, as previously described (15). Sham surgery was conducted by thoracotomy without LAD ligation. Cardiac rupture was identified by blood clotting in the chest cavity and ventricular wall tearing. ECG transmitters (Data Sciences International) were inserted into the abdominal cavity with subcutaneous electrodes in a lead I configuration. Upon recovery from surgery, continuous 24-hour recordings were started and continued for 1 week or sooner if mice died. At the end of each experiment, the data were extracted and analyzed for resting heart rate (defined as heart rate when activity was 0 ), heart rate response to activity, and arrhythmias. We defined spontaneous SND episodes as events of severe bradycardia, with heart rates of less than 200 beats per minute.

Mini-osmotic pump implantation. Micro-osmotic pumps (Alzet model 1004, $0.11 \mu \mathrm{l} / \mathrm{h}, 28$ days) containing MitoTEMPO $(700 \mu \mathrm{g} / \mathrm{kg} / \mathrm{d})$ were inserted subcutaneously into mice under anesthesia (ketamine/xylazine, 87.5/12.5 $\mathrm{mg} / \mathrm{kg}$ ), as reported previously (15).

Transthoracic echocardiography. We recorded transthoracic echocardiograms in conscious mice after MI, as previously described (65). Images were acquired and analyzed by an operator blinded to mouse genotype and treatment.

Human samples. Right atrial tissues were obtained from patients who had MI, with or without diabetes (Supplemental Table 3), as published previously (66).

SAN painting. Mice were painted within 1 week after STZ treatment, as previously described (17). Briefly, a mixture of $40 \%$ poloxamer, $1 \%$ trypsin, and $0.25 \%$ collagenase dissolved in PBS was added to equal volume of recombinant adenovirus-expressing plasmid for CaMKIIN-EGFP in an IRES vector or EGFP alone. Mice were anesthetized using ketamine/ xylazine $(87.5 / 12.5 \mathrm{mg} / \mathrm{kg}$, respectively). The gel was applied to the posterior surface of the junction of the superior vena cava and right atrium with a fine brush 3 times, with intervening periods of drying. Mice were sacrificed after 5 to 7 days, and hearts were harvested for ex vivo studies.

Langendorff perfusion and ex vivo ECG recording. Excised hearts were quickly mounted on a modified Langendorff apparatus, as described previously
(34). ECGs were continuously recorded with $\mathrm{Ag}^{+} / \mathrm{AgCl}$ electrodes positioned around the hearts in an approximate Einthoven configuration, as previously described (17). Langendorff-perfused hearts were stimulated with a pair of electrodes placed on the right atrium. Sinus node recovery time was measured after a 30 -second pacing train, with a basic cycle length of $100 \mathrm{~ms}$. For these studies, the sinus node recovery time was defined as the interval between the last stimulus in the pacing train and the onset of first sinus return beat. The CSNRT was calculated by subtracting the baseline cycle length from the sinus node recovery time.

SAN and atrial optical mapping. Optical mapping of spontaneous pacemaker activity and conduction velocity were performed as described previously (17).

SAN tissue isolation. Mice were sacrificed, and hearts were excised and placed in Tyrode's solution $\left(35^{\circ} \mathrm{C}\right)$, consisting of $140 \mathrm{mM} \mathrm{NaCl}, 5.0 \mathrm{mM}$ HEPES, $5.5 \mathrm{mM}$ glucose, $5.4 \mathrm{mM} \mathrm{KCl}, 1.8 \mathrm{mM} \mathrm{CaCl}_{2}, 1.0 \mathrm{mM} \mathrm{MgCl}_{2}$, with $\mathrm{pH}$ adjusted to 7.4 with $\mathrm{NaOH}$. The SAN region, delimited by the crista terminalis, atrial septum, and orifice of the superior vena cava, was dissected from the heart as previously described (17).

Immunofluorescence staining and SAN identification. The SAN tissues were cryopreserved and sectioned at $14-\mu \mathrm{m}$ thickness. We used anti-HCN4 antibodies to identify the SAN region (34). For all immunofluorescence staining, the slides were fixed and incubated with a blocking buffer and subsequently with the primary antibody to the protein of interest (rabbit anti-mouse ox-CaMKII and mouse anti-C-terminal CaMKII antibodies were developed by us) with HCN4 antibody (rat anti-human HCN4, Abcam), as previously described (17). For TUNEL staining, tissues were incubated using the In Situ Cell Death Detection Kit (Roche) for 1 hour at $37^{\circ} \mathrm{C}$, washed with PBS, and then mounted with DAPI Vectashield Medium (Vector Laboratories).

Immunoblotting. SAN tissues were homogenized in modified RIPA buffer (50 mM HEPES, pH 7.5, $150 \mathrm{mM} \mathrm{NaCl}, 5$ mM EDTA, 1\% v/v NP-40, and $0.5 \% \mathrm{w} / \mathrm{v}$ deoxycholate), containing a mixture of protease and phosphatase inhibitors. Equal amounts of protein were loaded on NuPAGE gels (Invitrogen) and transferred onto PVDF membranes (Bio-Rad). Blots were blocked, incubated in primary antibodies (ox-CaMKII and CaMKII) and subsequently with HRP-conjugated secondary antibodies, and finally detected using ECL reagent (Lumi-Light, Roche) as previously described (17). For quantification, band intensity was normalized to the entire Coomassie-stained gel using ImageJ software (NIH). Full uncut gels are shown in the Supplemental Material.

ROS detection. After cells or SAN sections were washed in warm PBS, DHE was applied for 30 minutes at $37^{\circ} \mathrm{C}$, as previously described (16). Fluorescence was detected with a laser scanning confocal microscope (Zeiss 510 and 710; excitation at $488 \mathrm{~nm}$ and detection at $585 \mathrm{~nm}$ by using a long-pass filter).

Caspase- 3 activity assay. SAN tissue was homogenized in lysis buffer consisting of $50 \mathrm{mM}$ Tris- $\mathrm{HCl}$, pH 7.5, $100 \mathrm{mM} \mathrm{KCl}, 1 \mathrm{mM}$ EDTA, 1 mM EGTA, $1 \mathrm{mM}$ DTT, $0.1 \mathrm{mM}$ PMSF, $0.5 \mathrm{mM}$ benzamidine, $20 \mathrm{mg} / \mathrm{l}$ leupeptin, 1,000 $\mathrm{mM}$ microcystin, $20 \mathrm{mM}$ sodium pyrophosphate, $50 \mathrm{mM} \mathrm{NaF}$, and $50 \mathrm{mM}$ sodium $\beta$-glycerophosphate, and total protein content was determined by the Bradford assay. Caspase-3 activity was determined by the EnzChek Caspase-3 Assay Kit (Invitrogen) as previously described (17).

Fibrosis detection and quantification. Masson's trichrome staining was used to assess fibrosis. We defined the SAN by HCN4-positive immunostaining (34). The images were imported into Image (NIH), and the pixels of blue staining were analyzed in the intranodal region (within the core SA node).

Isolated SAN cell action potential recording. SAN cells were isolated, and spontaneous single cell action potentials were recorded in current clamp mode using the perforated patch configuration at $36^{\circ} \mathrm{C}$, as described previously (34).

Neonatal cardiomyocyte culture. Hearts were digested with trypsin and collagenase II, incubated in media consisting of $40 \%$ F10, 40\% DMEM, $5 \%$ FBS, $5 \%$ ES for 24 hours, then in defined media for 24 hours as previously 
described (16), and subsequently exposed overnight to defined media with either glucose at low $(5 \mathrm{mM})$ or high concentrations $(33 \mathrm{mM})$ or mannitol $(33 \mathrm{mM})$ and cotreated with or without MitoTEMPO $(1 \mathrm{mM})$.

Mathematical modeling. Model geometry was represented as a 2-dimensional $400 \times 45$ rectangular grid with a spatial resolution of $40 \mu \mathrm{m}$. Cell types in different regions of the model were determined by histologically reconstructed sections through the rabbit RA, as described previously $(17,67,68)$. Cell loss in the SAN was simulated by randomly replacing a percentage of normal SAN cells (1\% for vehicle treated and $19 \%$ for STZ treated) with poorly coupled inexcitable cells. Detailed mathematical models were used to simulate central SANC, peripheral SANC, and atrial action potentials (Supplemental Table 4 and refs. 69, 70). Regional differences in cell size, coupling, and ion channel expression within the node were taken into account by making cell properties a function of space, following the previously described approach (68) (see Supplemental Equations). The 2-dimensional cable equation describing action potential propagation was solved using an alternating direction implicit method and a fixed time step of $0.005 \mathrm{~ms}$ (71). Initial conditions used for cells from different regions are provided in Supplemental Tables 5 and 6 . Eight seconds of spontaneous activity were simulated, and action potentials from different $\mathrm{SAN}$ regions were analyzed for activation pattern and rate. Computer code was written in $\mathrm{C}++^{+}$, compiled using Intel Composer XE 2011 for Linux, and executed on a Dell PowerEdge R515 server (Dual 6 core, 32 GB RAM running CentOS-6.2). Data from simulation of activation were visualized in MATLAB (R2012a).

Statistics. The survival analysis was performed using a log-rank test with Dunnett's test as a post-hoc study for multiple comparisons. All other statistical analyses were performed using an unpaired Student's $t$ test (2-tailed) or 1-way ANOVA with Dunnett's test (or Neuman-Keuls test as specified) for post-hoc study for multiple comparisons. $P<0.05$ was considered statistically significant. All values are expressed as mean \pm SEM.

Study approval. All animal procedures were approved by the Institutional
Animal Care and Use Committee at the University of Iowa. For human samples, the study was approved by the local ethics committee of Goettingen University, and each patient gave written informed consent.

\section{Acknowledgments}

We are grateful for discussions with K. Lamping, C. Benson, R. Felder, and F. Abboud (University of Iowa). We acknowledge the technical contributions of Chantal Allamargot at the Microscopy Core Facility (University of Iowa). We acknowledge the assistance of the University of Iowa Gene Transfer Vector Core for generating the CaMKIIN and EGFP virus. We thank Renee Goodfellow at the Gene Targeting Core Facility (University of Iowa) for her technical expertise in generating knockin mice. We thank R.E. Accaoui, D. Kraft, and $\mathrm{K}$. Zimmerman for their technical contributions to the mouse echocardiography. We thank M. Zimmerman for assistance with the statistical analysis. We acknowledge support by University of Iowa Cardiovascular Center Interdisciplinary Research Fellowship Training Grant from the NIH (to M. Luo) and the NIH (R01HL70250, R01HL079031, R01HL113001 and R01HL096652 to M.E. Anderson) as well as a grant (08CVD01) from the Fondation Leducq as part of the "Alliance for CaMKII Signaling in Heart." L.S. Maier is funded by the Deutsche Forschungsgemeinschaft (DFG) grant, Deutsches Zentrum für Herz-Kreislauf-Forschung (DZHK), and the Fondation Leducq.

Received for publication June 11, 2012, and accepted in revised form December 20, 2012.

Address correspondence to: Mark E. Anderson, 285 Newton Road, CBRB 2256, Iowa City, Iowa 52242, USA. Phone: 319.356.2745; Fax: 319.356.8608; E-mail: mark-e-anderson@uiowa.edu.
1. Knowler WC, et al. Reduction in the incidence of type 2 diabetes with lifestyle intervention or metformin. N Engl J Med. 2002;346(6):393-403.

2. Donahoe SM, et al. Diabetes and mortality following acute coronary syndromes. JAMA. 2007;298(7):765-775.

3. Barrett-Connor E, Orchard TJ. Insulin-dependent diabetes mellitus and ischemic heart disease. Diabetes Care. 1985;1:65-70.

4. Jacoby RM, Nesto RW. Acute myocardial infarction in the diabetic patient: pathophysiology, clinical course and prognosis. J Am Coll Cardiol. 1992;20(3):736-744.

5. Woodfield SL, et al. Angiographic findings and outcome in diabetic patients treated with thrombolytic therapy for acute myocardial infarction: the GUSTO-I experience. J Am Coll Cardiol. 1996;28(7):1661-1669.

6. Mak KH, et al. Influence of diabetes mellitus on clinical outcome in the thrombolytic era of acute myocardial infarction. GUSTO-I Investigators. Global Utilization of Streptokinase and Tissue Plasminogen Activator for Occluded Coronary Arteries. J Am Coll Cardiol. 1997;30(1):171-179.

7. Nesto RW, Zarich S. Acute myocardial infarction in diabetes mellitus: lessons learned from ACE inhibition. Circulation. 1998;97(1):12-15.

8. Zuanetti G, Latini R, Maggioni AP, Santoro L, Franzosi MG. Influence of diabetes on mortality in acute myocardial infarction: data from the GISSI-2 study. J Am Coll Cardiol. 1993;22(7):1788-1794.

9. Hill MF, Singal PK. Right and left myocardial antioxidant responses during heart failure subsequent to myocardial infarction. Circulation. 1997;96(7):2414-2420.

10. Palace VP, Hill MF, Farahmand F, Singal PK. Mobilization of antioxidant vitamin pools and hemody- namic function after myocardial infarction. Circulation. 1999;99(1):121-126.

11. Giacco F, Brownlee M. Oxidative stress and diabetic complications. Circ Res. 2010;107(9):1058-1070.

12. Di Filippo C, Cuzzocrea S, Rossi F, Marfella R, D'Amico M. Oxidative stress as the leading cause of acute myocardial infarction in diabetics. Cardiovasc Drug Rev. 2006;24(2):77-87.

13. Yusuf S, Dagenais G, Pogue J, Bosch J, Sleight P. Vitamin E supplementation and cardiovascular events in high-risk patients. The Heart Outcomes Prevention Evaluation Study Investigators. N Engl JMed. 2000;342:154-160.

14. Erickson JR, He BJ, Grumbach IM, Anderson ME. CaMKII in the cardiovascular system: sensing redox states. Physiol Rev. 2011;91(3):889-915.

15. Erickson JR, et al. A dynamic pathway for calcium-independent activation of CaMKII by methionine oxidation. Cell. 2008;133(3):462-474.

16. He BJ, et al. Oxidation of CaMKII determines the cardiotoxic effects of aldosterone. Nat Med. 2011;17(12):1610-1618.

17. Swaminathan PD, et al. Oxidized CaMKII causes cardiac sinus node dysfunction in mice.J Clin Invest. 2011;121(8):3277-3288

18. Rossini AA, Like AA, Chick WL, Appel MC, Cahill GF. Studies of streptozotocin-induced insulitis and diabetes. Proc Natl Acad Sci U S A. 1977;74(6):2485-2489.

19. Ling $\mathrm{H}$, et al. Requirement for $\mathrm{Ca} 2+/ \mathrm{calm}$ odulin-dependent kinase II in the transition from pressure overload-induced cardiac hypertrophy to heart failure in mice. $J$ Clin Invest. 2009;119(5):1230-1240.

20. Backs J, et al. The delta isoform of CaM kinase II is required for pathological cardiac hypertrophy and remodeling after pressure overload. Proc Natl Acad
Sci US A. 2009;106(7):2342-2347.

21. Zhang R, et al. Calmodulin kinase II inhibition protects against structural heart disease. Nat Med. 2005;11(4):409-417.

22. Abenavoli T, Rubler S, Fisher VJ, Axelrod HI, Zuckerman KP. Exercise testing with myocardial scintigraphy in asymptomatic diabetic males. Circulation. 1981;63(1):54-64

23. Izawa $\mathrm{K}$, et al. Impaired chronotropic response to exercise in acute myocardial infarction patients with type 2 diabetes mellitus. Jpn Heart J. 2003;44(2):187-199.

24. Hasslacher C, Wahl P. Diabetes prevalence in patients with bradycardiac arrhythmias. Acta Diabetol Lat. 1977;14(5-6):229-234.

25. La Rovere MT, Bigger JT Jr, Marcus FI, Mortara A, Schwartz PJ. Baroreflex sensitivity and heart-rate variability in prediction of total cardiac mortality after myocardial infarction. ATRAMI (Autonomic Tone and Reflexes After Myocardial Infarction) Investigators. Lancet. 1998;351(9101):478-484.

26. Solomon SD, et al. Sudden death in patients with myocardial infarction and left ventricular dysfunction, heart failure, or both. $N$ Engl J Med. 2005;352(25):2581-2588.

27. Jordaens L, Tavernier R. Determinants of sudden death after discharge from hospital for myocardial infarction in the thrombolytic era. Eur Heart J. 2001;22(14):1214-1225.

28. Oliva PB, Hammill SC, Edwards WD. Cardiac rupture, a clinically predictable complication of acute myocardial infarction: report of 70 cases with clinicopathologic correlations. J Am Coll Cardiol. 1993;22(3):720-726.

29. Curb JD, Rodriguez BL, Burchfiel CM, Abbott RD, Chiu D, Yano K. Sudden death, impaired glucose tolerance, and diabetes in Japanese American men. 
Circulation. 1995;91(10):2591-2595.

30. Aronson D, Rayfield EJ, Chesebro JH. Mechanisms determining course and outcome of diabetic patients who have had acute myocardial infarction. Ann Intern Med. 1997;126(4):296-306.

31. Malpas SC, Maling TJ. Heart-rate variability and cardiac autonomic function in diabetes. Diabetes. 1990;39(10):1177-1181.

32. Ferrer MI. The etiology and natural history of sinus node disorders. Arch Intern Med. 1982;142(2):371-372.

33. Gomes JA, Hariman RI, Chowdry IA. New application of direct sinus node recordings in man assessment of sinus node recovery time. Circulation. 1984;70(4):663-671.

34. Wu Y, et al. Calmodulin kinase II is required for fight or flight sinoatrial node physiology. Proc Natl Acad Sci U S A. 2009;106(14):5972-5977.

35. Chang BH, Mukherji S, Soderling TR. Characterization of a calmodulin kinase II inhibitor protein in brain. Proc Natl Acad Sci U S A. 1998;95(18):10890-10895.

36. Frustaci A, et al. Myocardial cell death in human diabetes. Circ Res. 2000;87(12):1123-1132.

37. Cai L, Li W, Wang G, Guo L, Jiang Y, Kang YJ. Hyperglycemia-induced apoptosis in mouse myocardium: mitochondrial cytochrome C-mediated caspase- 3 activation pathway. Diabetes. 2002;51(6):1938-1948.

38. Tomaselli GF, Barth AS. Sudden cardio arrest: oxidative stress irritates the heart. Nat Med. 2010;16(6):648-649.

39. Dikalova AE, et al. Therapeutic targeting of mitochondrial superoxide in hypertension. Circ Res. 2010;107(1):106-116.

40. Wilcox CS. Effects of tempol and redox-cycling nitroxides in models of oxidative stress. Pharmacol Ther. 2010;126(2):119-145.

41. Brownlee M. The pathobiology of diabetic complications: a unifying mechanism. Diabetes. 2005;54(6):1615-1625

42. Ozcan L, et al. Calcium signaling through CaMKII regulates hepatic glucose production in fasting and obesity. Cell Metab. 2012;15(5):739-751.

43. Fava S, Azzopardi J, Muscat HA, Fenech FF. Fac tors that influence outcome in diabetic subjects with myocardial infarction. Diabetes Care. 1993;16(12):1615-1618.

44. Howarth FC, Qureshi MA. Effects of carbenoxolone on heart rhythm, contractility and intracellular calcium in streptozotocin-induced diabetic rat. Mol Cell Biochem. 2006;289(1-2):21-29.

45. Kofo-Abayomi A, Lucas PD. A comparison between atria from control and streptozotocin-diabetic rats: the effects of dietary myoinositol. Br J Pharmacol.
1988;93(1):3-8.

46. Senges J, Brachmann J, Pelzer D, Hasslacher C, Weihe E, Kubler W. Altered cardiac automaticity and conduction in experimental diabetes mellitus. J Mol Cell Cardiol. 1980;12(12):1341-1351.

47. Boudina S, Abel ED. Diabetic cardiomyopathy revisited. Circulation. 2007;115(25):3213-3223.

48. Effect of intensive blood-glucose control with metformin on complications in overweight patients with type 2 diabetes (UKPDS 34). UK Prospective Diabetes Study (UKPDS) Group. Lancet. 1998;352(9131):854-865

49. Intensive blood-glucose control with sulphonylureas or insulin compared with conventional treatment and risk of complications in patients with type 2 diabetes (UKPDS 33). UK Prospective Diabetes Study (UKPDS) Group. Lancet. 1998;352:837-853.

50. Gerstein HC, et al. Effects of intensive glucose lowering in type 2 diabetes. $N$ Engl J Med. 2008;358(24):2545-2559.

51. Ray KK, et al. Effect of intensive control of glucose on cardiovascular outcomes and death in patients with diabetes mellitus: a meta-analysis of randomised controlled trials. Lancet. 2009;373(9677):1765-1772.

52 . Kosiborod M, et al. Glucometrics in patients hospitalized with acute myocardial infarction: defining the optimal outcomes-based measure of risk. Circulation. 2008;117(8):1018-1027.

53. Khattab M, Khader YS, Al-Khawaldeh A, Ajlouni K. Factors associated with poor glycemic control among patients with type 2 diabetes. J Diabetes Complications. 2010;24(2):84-89.

54. Ross SA, Tildesley HD, Ashkenas J. Barriers to effective insulin treatment: the persistence of poor glycemic control in type 2 diabetes. Curr Med Res Opin. 2011;3:13-20.

55. Capes SE, Hunt D, Malmberg K, Gerstein HC. Stress hyperglycaemia and increased risk of death after myocardial infarction in patients with and without diabetes: a systematic overview. Lancet. 2000;355(9206):773-778.

56 . Kosiborod M, et al. Admission glucose and mortality in elderly patients hospitalized with acute myocardial infarction: implications for patients with and without recognized diabetes. Circulation. 2005;111(23):3078-3086

57. Goyal A, et al. Prognostic significance of the change in glucose level in the first $24 \mathrm{~h}$ after acute myocardial infarction: results from the CARDINAL study. Eur Heart J. 2006;27(11):1289-1297.

58. Yu T, Robotham JL, Yoon Y. Increased production of reactive oxygen species in hyperglycemic conditions requires dynamic change of mitochondrial morphol- ogy. Proc Natl Acad Sci U S A. 2006;103(8):2653-2658.

59. Nishikawa $T$, et al. Normalizing mitochondrial superoxide production blocks three pathways of hyperglycaemic damage. Nature. 2000;404(6779):787-790.

60. Timmins JM, et al. Calcium/calmodulin-dependent protein kinase II links ER stress with Fas and mitochondrial apoptosis pathways. J Clin Invest. 2009;119(10):2925-2941.

61. Joiner ML, et al. CaMKII determines mitochondrial stress responses in heart. Nature. 2012;491(7423):269-73.

62. Zuanetti G, Latini R, Maggioni AP, Franzosi M, Santoro L, Tognoni G. Effect of the ACE inhibitor lisinopril on mortality in diabetic patients with acute myocardial infarction: data from the GISSI3 study. Circulation. 1997;96(12):4239-4245.

63. Moyé LA, et al. Uniformity of captopril benefit in the SAVE Study: subgroup analysis. Survival and Ventricular Enlargement Study. Eur Heart J. 1994;15(suppl B):2-8

64. Thompson MR, Chourey K, Froelich JM, Erickson BK, VerBerkmoes NC, Hettich RL. Experimental approach for deep proteome measurements from small-scale microbial biomass samples. Anal Chem. 2008;80(24):9517-9525.

65. Weiss RM, Ohashi M, Miller JD, Young SG, Heistad DD. Calcific aortic valve stenosis in old hypercholesterolemic mice. Circulation. 2006;114(19):2065-2069.

66. Sossalla $\mathrm{S}$, et al. Altered $\mathrm{Na}(+)$ currents in atrial fibrillation effects of ranolazine on arrhythmias and contractility in human atrial myocardium. J Am Coll Cardiol. 2010;55(21):2330-2342.

67. Dobrzynski $\mathrm{H}$, et al. Computer three-dimensional reconstruction of the sinoatrial node. Circulation. 2005;111(7):846-854.

68. Butters TD, et al. Mechanistic links between $\mathrm{Na}^{+}$ channel (SCN5A) mutations and impaired cardiac pacemaking in sick sinus syndrome. Circ Res. 2010;107(1):126-137.

69. Kurata Y, Matsuda H, Hisatome I, Shibamoto T. Regional difference in dynamical property of sinoatrial node pacemaking: role of na+ channel current. Biophys J. 2008;95(2):951-977.

70. Courtemanche M, Ramirez RJ, Nattel S. Ionic mechanisms underlying human atrial action potential properties: insights from a mathematical model. Am J Physiol. 1998;275(1 pt 2):H301-H321.

71. Joyner RW, Ramon F, Morre JW. Simulation of action potential propagation in an inhomogeneous sheet of coupled excitable cells. Circ Res. 1975;36(5):654-661.

72. Lin YC, Redmond L. Neuronal CaMKII acts as a structural kinase. Commun Integr Biol. 2009;2(1):40-41. 